\title{
An Overview of Three Promising Mechanical, Optical, and Biochemical Engineering Approaches to Improve Selective Photothermolysis of Refractory Port Wine Stains
}

\author{
Guillermo Aguilar, ${ }^{1}$ Bernard Choi, ${ }^{2,3}$ Mans Broekgaarden, ${ }^{4,5}$ Owen Yang, ${ }^{2,3}$ \\ Bruce Yang, ${ }^{2,3}$ Pedram Ghasri, ${ }^{2}$ Jennifer K. Chen, ${ }^{2}$ Rick Bezemer, ${ }^{6}$ J. Stuart Nelson, ${ }^{2,3}$ \\ Anne Margreet van Drooge, ${ }^{7}$ Albert Wolkerstorfer, ${ }^{7}$ Kristen M. Kelly, ${ }^{2}$ \\ and Michal Heger ${ }^{4,5}$
}

${ }^{1}$ Department of Mechanical Engineering, University of California, Riverside, CA, USA; ${ }^{2}$ Beckman Laser Institute and Medical Clinic, University of California, Irvine, CA, USA; ${ }^{3}$ Department of Biomedical Engineering, University of California, Irvine, CA, USA; ${ }^{4}$ Department of Experimental Surgery, Academic Medical Center, University of Amsterdam, Meibergdreef 9 , 1105 AZ Amsterdam, The Netherlands; ${ }^{5}$ Biochemistry of Membranes, Institute of Biomembranes, University of Utrecht, Utrecht, The Netherlands; ${ }^{6}$ Department of Translational Physiology, Academic Medical Center, University of Amsterdam, Amsterdam, The Netherlands; and ${ }^{7}$ Netherlands Institute for Pigment Disorders, Department of Dermatology, Academic Medical Center, University of Amsterdam, Amsterdam, The Netherlands

(Received 15 July 2011; accepted 6 October 2011; published online 21 October 2011)

Associate Editor Bahman Anvari oversaw the review of this article.

\begin{abstract}
During the last three decades, several laser systems, ancillary technologies, and treatment modalities have been developed for the treatment of port wine stains (PWSs). However, approximately half of the PWS patient population responds suboptimally to laser treatment. Consequently, novel treatment modalities and therapeutic techniques/strategies are required to improve PWS treatment efficacy. This overview therefore focuses on three distinct experimental approaches for the optimization of PWS laser treatment. The approaches are addressed from the perspective of mechanical engineering (the use of local hypobaric pressure to induce vasodilation in the laser-irradiated dermal microcirculation), optical engineering (laser-speckle imaging of post-treatment flow in laser-treated PWS skin), and biochemical engineering (light- and heat-activatable liposomal drug delivery systems to enhance the extent of post-irradiation vascular occlusion).
\end{abstract}

Keywords-Vascular malformations, Dermis, Suboptimal therapeutic response and lesional recalcitrance, Hypobaric pressure, Suction cup, Blood flow, Laser speckle imaging, Site-specific pharmaco-laser therapy, Liposomal drug delivery system.

Address correspondence to Michal Heger, Department of Experimental Surgery, Academic Medical Center, University of Amsterdam, Meibergdreef 9, 1105 AZ Amsterdam, The Netherlands. Electronic mail: M.Heger@amc.uva.nl

The work by author Guillermo Aguilar was in part performed at Centro de Investigación y Estudios Avanzados (CINVESTAV) del Instituto Politécnico Nacional, campus Querétaro, México during sabbatical leave.

\section{INTRODUCTION}

Port wine stains (PWSs) are congenital vascular lesions characterized by hyperdilated microvasculature predominantly in the upper dermis. Due to the high blood content in the hyperdilated blood vessels, the affected region of the skin appears red-to-purple (Fig. 1a). The color intensity of the lesions typically worsens with age and, when left untreated, approximately two-thirds of the lesions develop nodular components and facial deformity. ${ }^{44,55,59,106}$ Apart from being a cosmetic problem with often considerable psychosocial ramifications for the patient, ${ }^{86}$ some lesions present with ocular and neurological disorders such as glaucoma and seizures, respectively. ${ }^{136}$ Consequently, a strong need exists to optimally treat PWSs.

The standard treatment of PWSs entails laser irradiation of the affected portions of the skin (Fig. 1b) in accordance with the theory of selective photothermolysis (SP). ${ }^{7}$ Laser irradiation leads to photocoagulation of the hyperdilated blood vessels, which in turn results in their removal and subsequent clearance of the PWS. ${ }^{64}$ Clinical studies have shown that complete photocoagulation of the vascular lumen is associated with complete lesional clearance, and that partial photocoagulation is associated with suboptimal responsiveness. ${ }^{48,60,72,137}$ Therefore, the key to effectively treating recalcitrant lesions is the complete (photo)occlusion of PWS vasculature. 
(a)

(b)
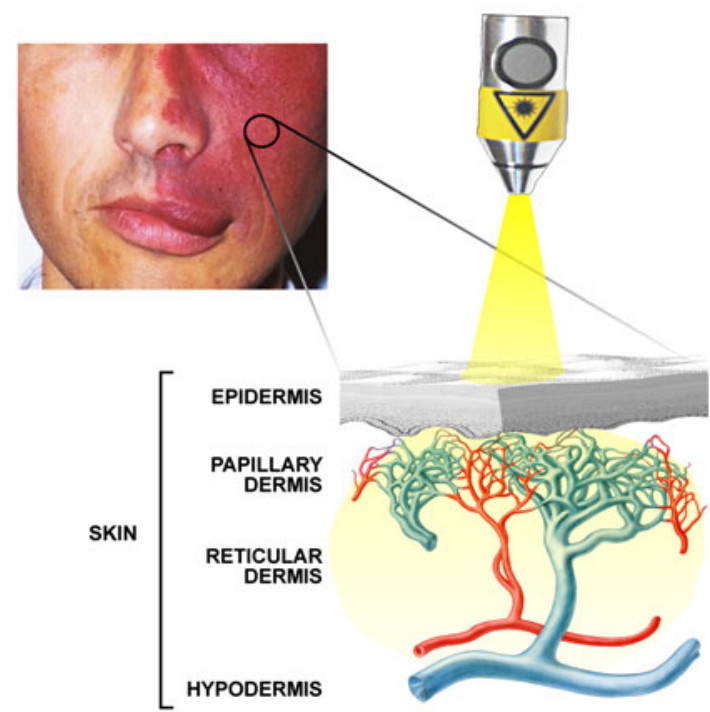

(c) DEGREE OF PWS CLEARANCE:
$0-25 \%$

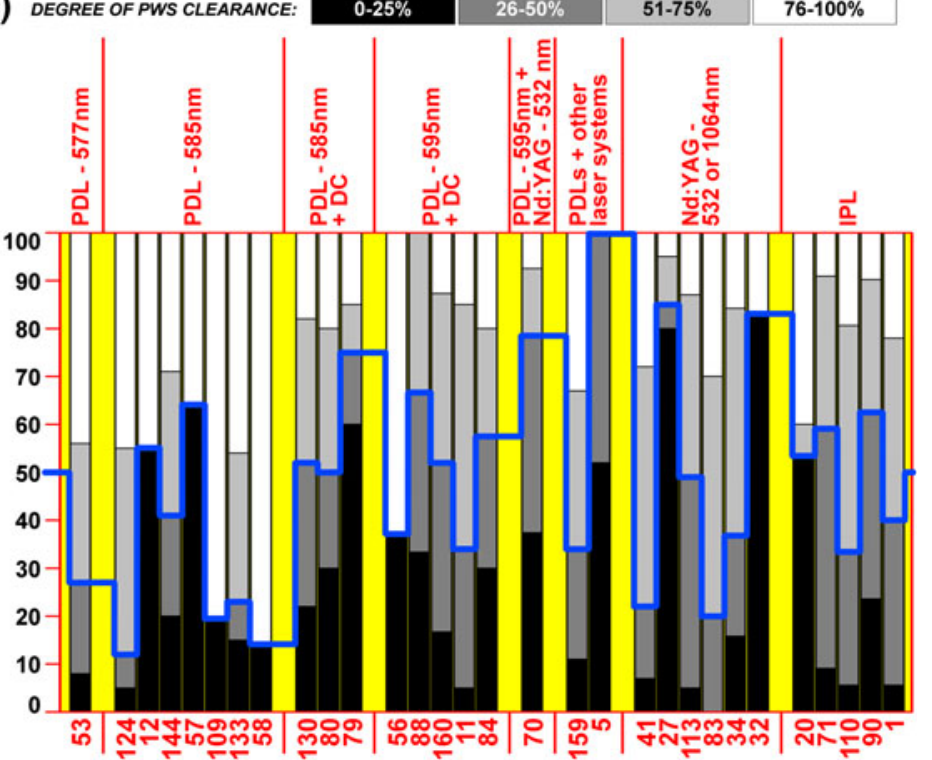

FIGURE 1. (a) Image of a facial PWS, accompanied by soft tissue overgrowth in the mouth area. The encircled portion of the patient's PWS is enlarged in (b) and presented as a cross section. The various layers of the skin are indicated. The dermis is replete with microcirculation, whereby the arterial segment of the vasculature is depicted in red and the venular segment in blue. Hyperdilation of blood vessels in PWSs prevails predominantly in the venular segment. PWSs are treated non-invasively with lasers (probe), most frequently with pulsed dye lasers (PDLs) in the 577-600 nm range or Nd:YAG lasers (1064 $\mathrm{nm}$ or its second harmonic, $532 \mathrm{~nm}$ ) because of the preferential absorption by hemoglobin. The laser beam significantly diverges in the skin because of scattering. In (c), a summary is provided of clinical studies published between 1990 and 2010, in which the clinical outcome was scored in accordance with the legend ("degree of PWS clearance"). The $y$-axis indicates the percentage of patients, the $x$-axis lists the clinical studies by their respective reference. The study outcomes are presented vertically, where each bar represents a grayscale-coded stratum according to the legend. The studies were categorized by laser modality, separated by the vertical red lines and yellow tabs in the chart. The blue line corresponds to the percentage of patients at which the median treatment outcome was reached, i.e., the percentage of patients in which $0-50 \%$ clearance (black + dark gray bars) was achieved. This line reflects the treatment efficacy over the sampling period, showing that improvement in PWS therapy is needed and that the degree of lesional clearance does not necessarily depend on the laser modality. Studies with deviant scoring system: references $15,17,24(0-75 \%$ clearance $=$ black, $76-100 \%$ clearance $=$ white $)$, references 18,20 , and $37(0-50 \%$ clearance $=$ black, $51-100 \%$ clearance $=$ white $)$. PDL = pulsed dye laser, DC = dynamic cooling, IPL = intense pulsed light. Image of skin and vascular anatomy courtesy of Ms. Libuše Markvart.

Unfortunately, the clinical reality is that laser therapy is effective in approximately half of PWS patients (Fig. 1c). 1,5,11,12,20,27,32,34,41,53,56-58,70,71,79,80,83 84,88,90, 109,110,113,124,130,133,144,159,160 This overview therefore focuses on the causes of the therapeutic recalcitrance and presents several experimental engineering approaches, including (1) alterations in local hemodynamics, (2) concomitant laser treatment and dermovascular imaging, and (3) site-specific pharmaco-laser therapy, with which the therapeutic efficacy of recalcitrant PWSs may be improved.

\section{ENDOVASCULAR LASER-TISSUE INTERACTIONS}

\section{Selective Photothermolysis}

Selective photothermolysis is a non-invasive laser treatment modality that relies on the conversion of radiant energy to heat by (oxy)hemoglobin and the ensuing coagulation of blood and necrosis of (peri)vas- cular tissue resulting from thermal diffusion (Fig. 2a). ${ }^{7,15,19,21,22,107,150}$ By irradiating skin at a wellabsorbed wavelength and sufficient irradiance (the amount of photon energy incident on a unit area of tissue per unit time, expressed in $\mathrm{W} / \mathrm{cm}^{2}$ ), supracritical temperatures $\left(>70{ }^{\circ} \mathrm{C}\right)$ can be generated in the vessel lumen and confined spatially if the pulse duration is kept within the thermal relaxation time of the target blood vessel or, in case of large-diameter vasculature, the volume of blood in which the radiant energy is absorbed. Thermal relaxation time is defined as the time required for heated matter to lose $50 \%$ of its peak thermal energy through thermal conductivity. ${ }^{7,147}$ The ideal pulse duration for blood vessels of $20-150 \mu \mathrm{m}$ has been shown to range from 1 to $10 \mathrm{~ms} .{ }^{39}$ Normal-sized capillaries $(4-6-\mu \mathrm{m}$ inner diameter) and post-capillary venules $(8-26-\mu \mathrm{m}$ inner diameter $),{ }^{24}$ which have relatively short thermal relaxation times and a smaller volume-to-surface ratio (thermal mass), therefore remain spared during longer pulse durations, as heat diffusion from these vessels precludes the generation of denaturing temperatures. 
(a) SELECTIVE PHOTOTHERMOLYSIS

1.
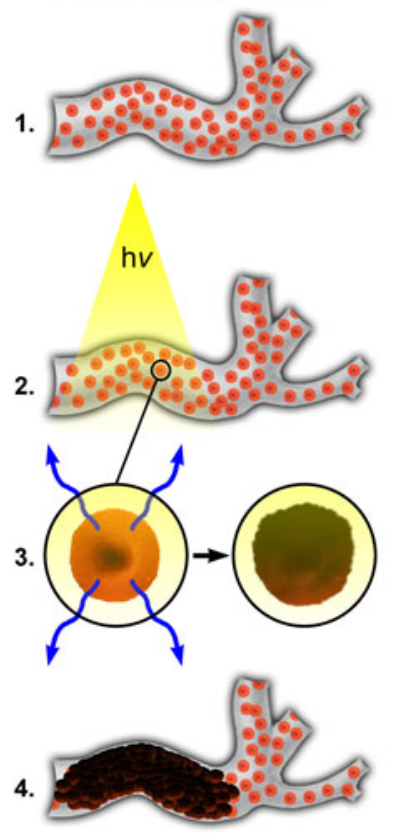

4. (b) DIFEREETTAL RESPONSES TO SELECTIVE PHOTOOHERMOLYSIS

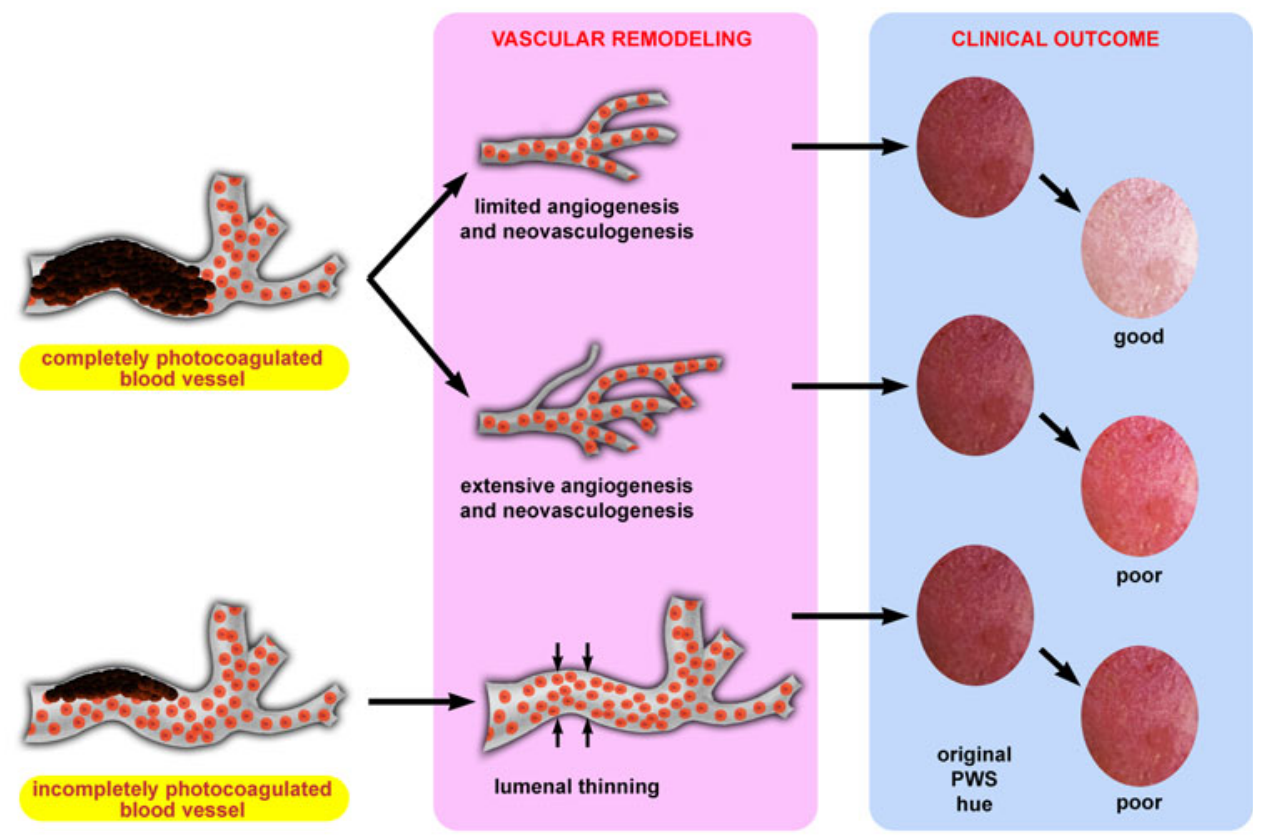

FIGURE 2. (a) Endovascular laser-tissue interactions in relation to SP. When an erythrocyte-filled blood vessel (1, red spheres = erythrocytes) is irradiated with a laser $(2, h v)$ at a wavelength that is predominantly absorbed by hemoglobin in the erythrocyte (3, left panel), the radiant energy is converted to heat $(3$, blue arrows left panel) that subsequently diffuses away from the erythrocyte. The biomolecules (DNA, proteins, and lipids) in the regions of supracritical heating $\left(>70^{\circ} \mathrm{C}\right)$ undergo thermal denaturation (photocoagulation), as a result of which cells and structures lose their native physicochemical properties (3, right panel). Blood undergoing photocoagulation forms a thermal coagulum-an amorphous clump of damaged and agglutinated erythrocytes and plasma constituents-with which the vascular lumen becomes occluded (4). The extent to which PWS vasculature is photocoagulated during laser treatment essentially dictates the biological response to SP, outlined in (b). The putative contention is that complete photo-occlusion (top pathway) most often results in vascular remodeling characterized by removal of the thermally afflicted vasculature followed by limited angiogenesis and/or neovasculogenesis. These processes typically result in good clinical clearance inasmuch as the dermal blood content is considerably reduced after the vascular remodeling phase ("clinical outcome" panel, illustrating the changes in skin color before (left) and after (right) treatment). Alternatively, when angiogenic/neovasculogenic remodeling following laser treatment is extensive, sufficient reduction in dermal blood volume is hampered and only mild clearance is achieved (middle pathway). In other instances, particularly in refractory PWSs, light penetration is insufficient to induce complete photocoagulation of the vascular lumen, resulting in partial occlusion of the target vessel by a thermal coagulum (bottom pathway). During the remodeling phase, the thermal coagulum is either removed by the reticuloendothelial system or becomes part of the vascular wall, leading to luminal thinning (small opposing arrows). This damage profile is associated with minimal reduction in dermal blood volume and hence poor clinical outcome.

In larger vessels such as PWS vasculature, the laserinduced production of supracritical temperatures leads to thermal necrosis of the vascular wall and vasoocclusion by thermolysed and agglutinated erythrocytes. ${ }^{19,21,48,72,137,138}$ The putative contention is that the photocoagulated vessels are removed by the reticulo-endothelial system and replaced by normalsized capillaries through vascular remodeling (Fig. 2b). ${ }^{64,76,116}$ Replacement of hyperdilated vessels with lower blood volume-containing capillaries is associated with a reduction in PWS redness (Fig. 2c), which marks the therapeutic end point. Clinically, complete photocoagulation of the vascular lumen is associated with well-responding lesions, ${ }^{48}$ corresponding to approximately $40 \%$ of the cases. ${ }^{60}$ In contrast, moderately responding $(20-46 \%)$ and refractory (14-40\%) PWSs have an acute post-treatment damage profile characterized by varying degrees of partially photocoagulated vessels with semi-obstructive thermal coagula. $48,72,137$

\section{Causes of Therapeutic Recalcitrance}

The efficacy of SP depends on a combination of inevitable intrinsic factors, including epidermal pigmentation, ${ }^{36,80,146,153}$ optical shielding by blood and superimposed vessels, ${ }^{47,48,72,92,117,137}$ and vascular anatomy and morphology. ${ }^{47,48,72,117,149,154}$ In general, treatment efficacy correlates negatively with increased melanin content, vascular density and superimposition, and vessel diameter and depth, given that the prominence of these factors is inversely proportional to the optical penetration depth. Inasmuch as the optical penetration depth, and thus the fluence rate $(\phi$, the 
amount of photon energy incident on a unit area inside tissue per unit time, expressed in $\mathrm{W} / \mathrm{cm}^{2}$ ) attenuates with depth in accordance with Beer's law, scattering, and (specular) reflection, ${ }^{114,149}$ the volumetric heat production $\left(\phi \cdot \mu_{\mathrm{a}}\right.$, where $\mu_{\mathrm{a}}$ is the absorption coefficient) in blood vessels located beyond a certain depth will be inadequate for the complete photocoagulation of the vascular lumen.

Port wine stain vessels have been found in the reticular plexus up to a depth of $3.7 \mathrm{~mm},{ }^{139,145}$ albeit most of the ectatic vasculature is located within $\sim 0.6 \mathrm{~mm}$ from the basal membrane. ${ }^{14,72}$ Theoretically, complete lesional clearance can only be accomplished when the optical penetration depth equals or exceeds the depth up to which the ectatic vasculature is responsible for the visual appearance of the PWS. Mathematical modeling predicted this depth to be $0.6-$ $0.9 \mathrm{~mm}$ at which hyperdilated vessels contribute to the skin discoloration. ${ }^{85,153}$ Histological studies have revealed that photocoagulation occurs to a depth of $\sim 0.65 \mathrm{~mm}$ in human skin using $585-\mathrm{nm}$ wavelength and 0.45 -ms pulse duration, with a mean \pm SD depth of $0.37 \pm 0.17 \mathrm{~mm} .^{48,72}$ At these laser parameters (and a radiant exposure of $6.5 \mathrm{~J} / \mathrm{cm}^{2106}$ ), complete coagulation of the vessel lumen occurs in superficial vessels up to approximately $150 \mu \mathrm{m}$ in diameter. ${ }^{72}$ Larger and deeper vessels will therefore remain (partly) patent, as has been corroborated histologically and numerically. ${ }^{92,152}$ In the context of the model predictions, these findings explain why some PWSs are nonresponsive to treatment at the abovementioned laser parameters based on depth alone.

With respect to optical penetration, it is not surprising that the difference between the irradiance and the fluence rate becomes even more pronounced with depth in densely vascularized areas ${ }^{92,114,155}$ and in skin types with a higher melanin concentration ${ }^{72,154}$ and dermal blood volume. ${ }^{154}$ Consequently, incomplete photocoagulation of target vessels may result from the generation of subcritical isotherms $\left(<70^{\circ} \mathrm{C}\right)$ because of inhomogeneous photon deposition in the lumen (as is the case with large diameter vessels), or may be forestalled altogether by insufficient heat production across the entire vessel diameter, such as in deeply situated or optically shielded vessels. Differences in intralumenal fluence rates due to the abovementioned factors therefore have a profound effect on the acute and chronic tissular responses (Fig. 2b), and thus lesional clearance.

\section{HEMODYNAMIC ALTERATIONS IN THE DERMIS}

Rather than attempting to bring more light to the blood, which has yielded little clinical improvement over the past 20 years (Fig. 1c), more blood could be brought to the already available light to optimize the extent of laser-mediated photocoagulation. Several premises lie at the basis of systematically inducing hemodynamic alterations in the PWS dermis through the local application of hypobaric pressure. First, the venules in mild red or faint PWSs have an average diameter of $10-30 \mu \mathrm{m},{ }^{115}$ and hence a thermal relaxation time that is shorter than the $0.5-1.5$-ms pulses emitted by most pulsed dye lasers. ${ }^{7}$ Consequently, the target vessels are difficult to photocoagulate by SP because the volumetric heat production is insufficient to adequately heat the entire vascular lumen. Second, smaller vessels have a lower erythrocyte concentration and thus less chromophore to generate supracritical temperatures required for complete photocoagulation of the vascular lumen. A recent clinical study, ${ }^{18}$ where PWS treatment was performed with an intense pulse light system, revealed that it was difficult to photocoagulate vessels of $<60 \mu \mathrm{m}$ in diameter at radiant exposures of $<30 \mathrm{~J} / \mathrm{cm}^{2}$. These findings were attributed to insufficient heat generation in the vascular lumen because of the low hemoglobin concentrations in the small-diameter vessels. Third, small-diameter vessels also have a lower thermal mass, as a result of which heat dissipation into perivascular tissue (i.e., cooling) proceeds faster than in larger vessels. Accordingly, the augmentation of venular diameters through the application of hypobaric pressure will temporarily synchronize the thermal relaxation time of these vessels with the standardized pulse durations employed and concomitantly increase intravascular chromophore content and thermal mass.

\section{Proposed Mechanism of Vacuum-Induced Vasodilation}

The vacuum-induced pressure gradient across a vessel is not the main cause of its dilation, but rather the body's physiological response to locally applied pressure. Vasoactivity in the cutaneous microcirculation is partly controlled by the body's systemic mechanisms, i.e., by cardiac output, the amount of resistance imposed by the arteries/arterioles, and by varying the amount of blood that is pooled within the veins/venules. ${ }^{35}$ The latter two aspects are in turn regulated by vasoactive substances secreted into the circulation and by vasomotor nerves that innervate the mural tissue of arterioles.

Local vasoactivity of arterioles and venules depends on the balance between plasma concentrations of vasodilators [nitric oxide (NO), prostacyclin, carbon monoxide], and vasoconstrictors (endothelin-1, thromboxane $\mathrm{A}_{2}$ ). These compounds are secreted by proximal cells such as endothelial cells that line the lumen of blood vessels. ${ }^{52}$ Of these, NO acts directly on pericytes 
in the vascular wall and causes these cells to relax. Endothelial cells further respond to stimuli such as flow changes, stretching, and shear stress, whereby the latter triggers the release of NO. ${ }^{33,40,111}$ When suction is applied to a localized region on the skin surface, it is believed that the endothelial cells exposed to the mechanical strain and stress release NO that in turn triggers the vasodilation.

\section{Physical and Tissular Effects of Hypobaric Pressure on Skin}

A hypobaric pressure-modulatable device has been developed to locally increase vascular diameters in PWS skin (Fig. 3a). Several investigations on this device have employed numerical models in combination with experimental work to characterize and validate hypobaric pressure-related predictions and hypotheses. With respect to the mechanical effects of local vacuum on skin, Childers et al. ${ }^{29}$ developed a macroscopic model comprising classical structural mechanics equations solved by finite element methods to compute the extent of lateral stretching and axial displacement of PWS skin in a suction cup. The predictions were juxtaposed to experimental data obtained by video imaging of vacuum-deformed skin, demonstrating excellent agreement between the computed and measured deformations (Fig. 3b). ${ }^{29}$

The stress-strain field computations by Childers et al. ${ }^{29}$ subsequently served as a boundary condition for the development of a model to determine the microscopic thinning of the epidermis, the axial displacement of a simulated blood vessel, and its dilation. The model predicted (1) epidermal thinning of $6 \mu \mathrm{m}$ at maximum hypobaric pressure $(50 \mathrm{kPa}, 381 \mathrm{mmHg})$, (2) a $2.5-\mu \mathrm{m}$ displacement of a typical blood vessel at $250 \mu \mathrm{m}$ below the skin surface, and (3) only a 5\% dilation of this vessel at the indicated pressure.

The extent of vasodilation as determined by Childers et al. ${ }^{29}$ was rather disproportionate to the underlying hypothesis of vacuum-induced vessel dilation and in contrast with subsequent theoretical/ empirical findings. For instance, visible reflectance spectrometry measurements on vacuum-treated PWS skin provided indirect evidence for vacuum-induced changes in vascular diameter that, based on a light diffusion approximation model, encompassed a twofold dilation and a $10 \%$ increase in the blood volume fraction (BVF) at $-50 \mathrm{kPa}(381 \mathrm{mmHg}){ }^{134}$ The $5 \%$ vessel dilation predicted by Childers' microscopic mode $^{29}$ corresponded to a mere $0.3 \%$ increase in the $\mathrm{BVF}$, which lies below the limit of detection of visible reflectance spectrometry. It was further shown that, during the first $0.5 \mathrm{~s}$ of vacuum application, the skin underwent a reduction in BVF and vessel diameter
$\left(D_{\mathrm{v}}\right)$, while the bulk tissue was being deformed by the suction. After this time interval, however, both BVF and $D_{\mathrm{v}}$ rose from 1.3 to $10.5 \%$ and from 9.5 to $20.0 \mu \mathrm{m}$, respectively, reaching a plateau after $\sim 9 \mathrm{~s}$ (Fig. 3c). ${ }^{3,29,49}$ Moreover, the kinetics of BVF and $D_{\mathrm{v}}$ during the application of hypobaric pressure were characterized by a fast rate increase followed by a slower rate increase. The initial increase in BVF and $D_{\mathrm{v}}$ was likely driven by predominately biochemically mediated muscle relaxation, whereas the subsequent (lower rate) increase in $\mathrm{BVF}$ and $D_{\mathrm{v}}$ was likely driven by the mechanical strain applied to the vessel wall after the smooth muscle cells had fully relaxed.

\section{Effects of Hypobaric Pressure on Cutaneous Laser Treatment}

Hypobaric pressure does not only induce vasodilation but exerts several other effects that are beneficial for the laser treatment of PWSs. Franco et al. ${ }^{49} \mathrm{em}-$ ployed a numerical model of light and heat diffusion that yielded three important aspects of laser-tissue interactions at hypobaric pressure. First, hypobaric pressure causes less radiant energy to be absorbed in the epidermis because the stretching of the skin reduces epidermal thickness and the concentration of melanin per unit area. Second, more light is delivered to an increased blood volume, as a result of which higher intralumenal temperatures can be generated. The extent of volumetric heat production in the lumen is proportional to the amount of hypobaric pressure applied (Fig. 3d). Third, the higher energy deposition in a larger blood volume was found to correlate to the induction of higher temperatures in all vessel sizes, although the temperature increases in $10-30-\mu \mathrm{m}$ diameter vessels were more significant (Figs. 3e and $3 \mathrm{f})$. This exacerbates the degree of irreversible thermal damage to small, recalcitrant PWS vasculature, while keeping other blood vessels relatively intact.

Corroboratively, Yeo et al. ${ }^{163}$ demonstrated in ex vivo porcine skin that the peak intensity of the incident laser photon density increased 3.6-fold when used in combination with hypobaric pressure at $30 \mathrm{kPa}$ $(254 \mathrm{mmHg})$. These effects were attributed to tissue thickness reduction and water content loss as a result of the mechanical stretching/compression of skin. The vacuum-induced deformation of skin further decreases the effective distance between adjacent chromophores (i.e., blood vessels) as well as the distance between elastin layers. Those authors therefore hypothesized that these phenomena improve refractive index matching and that, consequently, the increase in laser photon density in tissue could potentially improve the therapeutic efficacy for PWS. 
(a)

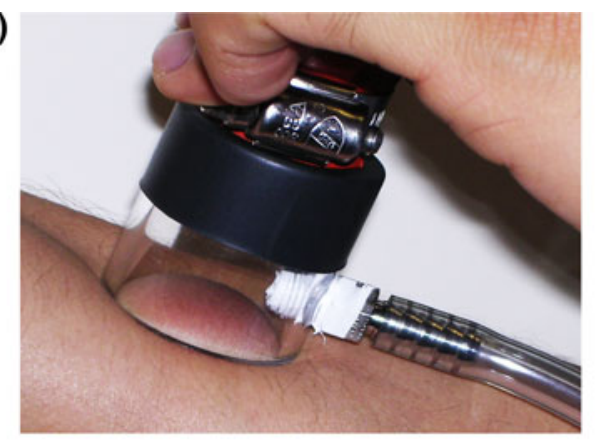

(c)
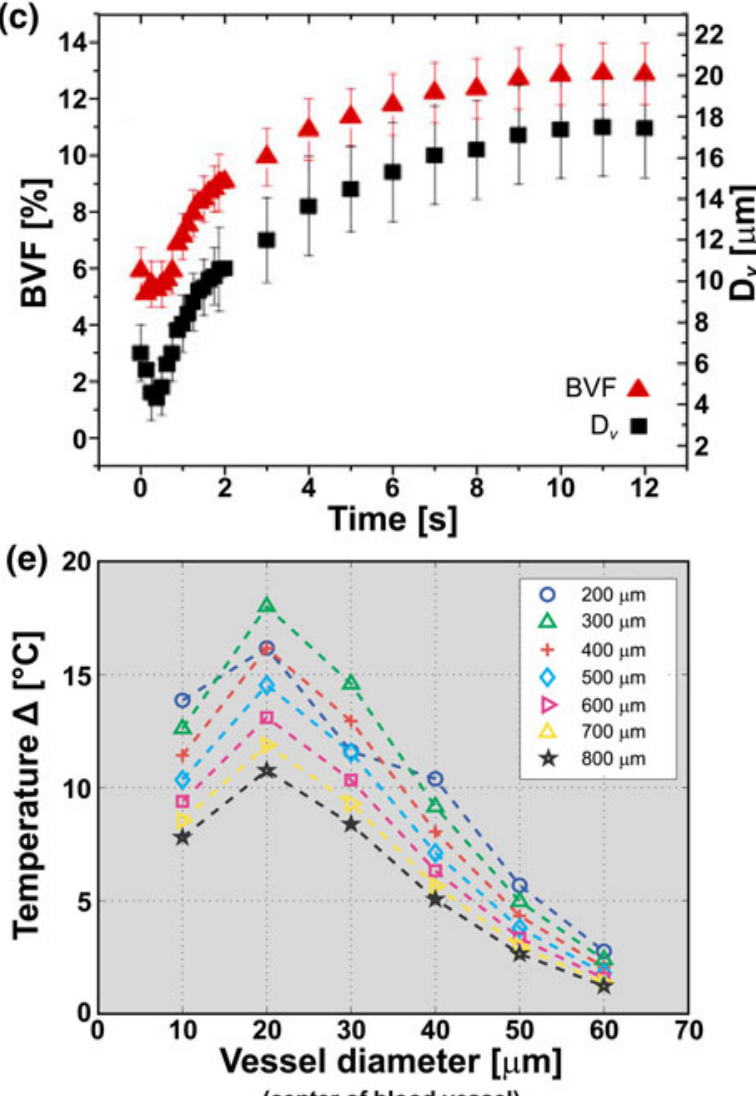

(b)

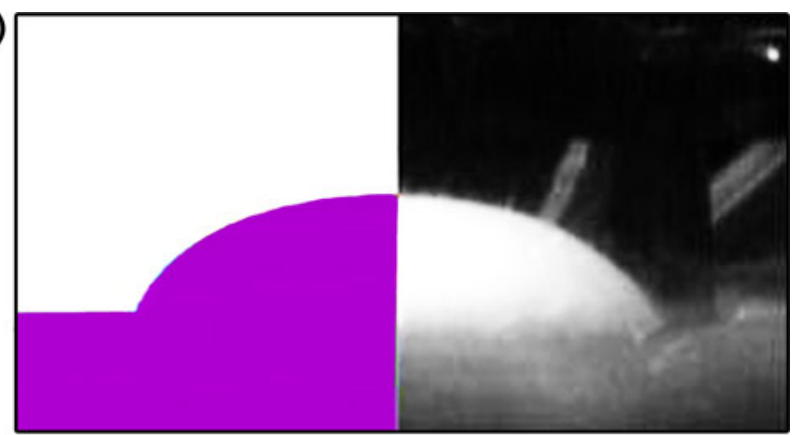

(d)

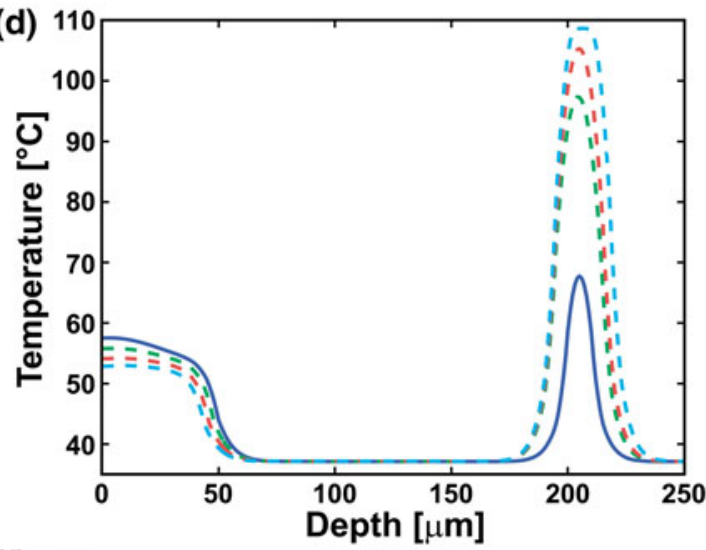

(f)

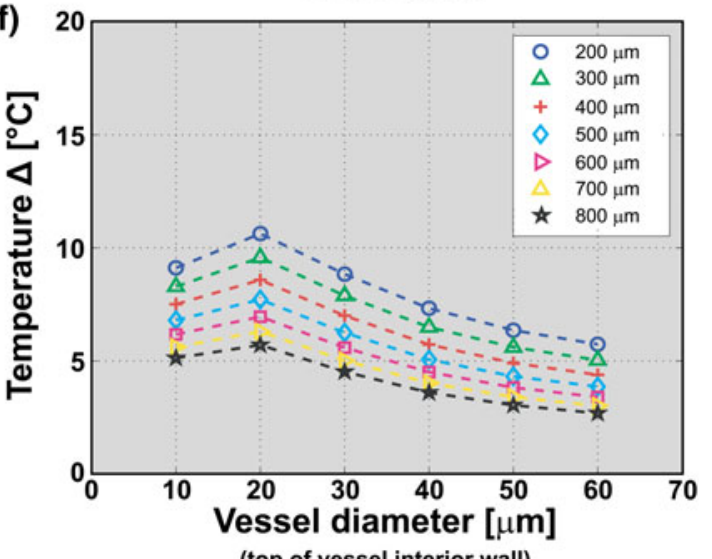

FIGURE 3. (a) An example of a suction cup that is used to induce local hypobaric pressure on the skin. (b) Image of bulk tissue deformation on the forearm of a subject exposed to $34 \mathrm{kPa}(247 \mathrm{mmHg})$ of hypobaric pressure (right panel) and the skin deformation profile as computed by a structural mechanics model ${ }^{29}$ at the same hypobaric pressure (left panel). (c) Small-scale numerical model and visible reflectance spectroscopy results reflecting changes in blood flow velocity (BVF) and average vessel diameter $\left(D_{v}\right)$ as a function of time during the application of 50-kPa hypobaric pressure on the palm of a 24-year-old male. Data modified from Aguilar et al. $^{3}$ (d) Numerical analysis of temperatures ( $y$-axis) generated at the end of a laser pulse (585-nm wavelength, 0.45-ms pulse duration, $1-\mathrm{J} / \mathrm{cm}^{2106}$ radiant exposure, $10-\mathrm{mm}$ spot size) at different dermal depths ( $x$-axis) in Fitzpatrick type II skin, plotted for increasing local hypobaric pressures. A 10- $\mu \mathrm{m}$-diameter blood vessel was positioned at $200 \mu \mathrm{m}$ below the skin surface. Data taken from Franco et al. ${ }^{49}$ Differences in blood (e) and vessel wall temperatures ( $f$ ) at the end of the laser pulse (585-nm wavelength, 0.45ms pulse duration, $1-\mathrm{J} / \mathrm{cm}^{2}$ radiant exposure, $10-\mathrm{mm}$ spot size) at hypobaric and atmospheric pressures, plotted as a function of vessel diameter for different vessel depths (legend). Fitzpatrick skin type 2 was modeled. Data taken from Franco et al. ${ }^{49}$

\section{Effects of Hypobaric Pressure on Cryogen Spray Cooling Dynamics}

Epidermal pigmentation (melanin) constitutes the most important fluence-limiting factor in the laser treatment of PWS. Melanin absorption at the clinically employed pulsed dye laser wavelengths is greater than hemoglobin absorption, as a result of which a balance has to be struck between optical penetration depth and the maintenance of subcritical temperatures at the stratum basale - the deepest epidermal layer containing the melanocytes. This balance is governed by the employed laser settings (wavelength, pulse duration, 
and irradiance), whereby too high fluences induce considerable photocoagulation of PWS vasculature but thermally afflict the epidermis, while too low fluences result in incomplete photocoagulation but preserve epidermal integrity. By the application of epidermal cooling technologies, the balance can be shifted in favor of the optical penetration depth by controlling the extent of heat generation at the stratum basale during a laser pulse.

The most common epidermal cooling techniques include 1,1,1,2-tetrafluoroethane (R134a)-mediated cooling, commonly referred to as cryogen spray cooling (CSC), contact cooling, ${ }^{6}$ and cold air cooling. ${ }^{122}$ In the mid-90s, several proof-of-concept studies demonstrated that CSC was effective in protecting normal skin $^{9}$ and PWS skin epidermis ${ }^{9,105}$ from high-irradiance laser pulses. Subsequent infrared radiometric experiments showed that CSC-induced temperature reductions can be confined to the upper $\sim 200 \mu \mathrm{m}$ of skin (i.e., the average thickness of facial epidermis), ${ }^{8}$ allowing epidermal cooling without affecting the extent of vessel photocoagulation. Also, the heat flux and total energy removed per unit skin surface area were shown to be at least doubled when using CSC vs. alternative cooling methods such as contact cooling. ${ }^{8}$ Consequently, CSC has become the most studied and implemented cooling technique in dermatological laser applications, particularly for shallow targets such as PWS vasculature.

The use of hypobaric pressure not only entails the previously mentioned advantages for PWS laser therapy ("Effects of Hypobaric Pressure on Cutaneous Laser Treatment" section) but also facilitates a more efficacious epidermal cooling protocol in a number of ways. First, recent studies evinced that, under hypobaric pressures, evaporating sprays such as R134a have a larger adiabatic expansion, ${ }^{2}$ which means that their saturation or evaporating temperature is reduced under vacuum conditions. With respect to R134a, the evaporation temperature at atmospheric pressure is roughly $-26{ }^{\circ} \mathrm{C} .{ }^{134}$ The evaporation temperature of R134a can be reduced in a hypobaric pressure-dependent fashion (Fig. 4a), up to $15^{\circ} \mathrm{C}$ at $-51 \mathrm{kPa}(381 \mathrm{mmHg})$ vs. atmospheric pressure, by releasing the CSC spurt within a small vacuum chamber or suction cup. This was further elaborated by Aguilar et al. ${ }^{2}$ through systematic measurements of skin phantom surface temperature variations and heat fluxes for spurts delivered at increasing hypobaric pressures. The extent of surface cooling was proportional to the extent of hypobaric pressure at equivalent CSC settings (Fig. 4b), with the difference in the minimum surface temperature reaching as high as $20^{\circ} \mathrm{C}$ in the $67-\mathrm{kPa}(508 \mathrm{mmHg})$ hypobaric pressure group vs. the atmospheric pressure group. Similarly, the degree of CSC-mediated heat extraction from the skin phantoms correlated positively with the magnitude of hypobaric pressure (Fig. 4c). The observed effects are attributable to the larger pressure-induced expansion of the CSC spurt (see next paragraph) and larger temperature differential between the cryogen and skin phantom surface temperature with increasing hypobaric pressures (Fig. 4a).

In terms of spray atomization, i.e., the fragmentation of fluids into drops, it was demonstrated that higher droplet velocities are associated with better atomization and, consequently, a more homogeneous cryogen spray deposition due to the larger pressure differential across the spray nozzle. ${ }^{2}$ Light reflection experiments revealed that the "jet length," or the distance a core jet of cryogen travels before atomizing into smaller droplets, was significantly reduced at lower pressures in the expansion chamber (Fig. 5a).

Third, under atmospheric conditions the spray impingement on the skin may produce an indented (a)

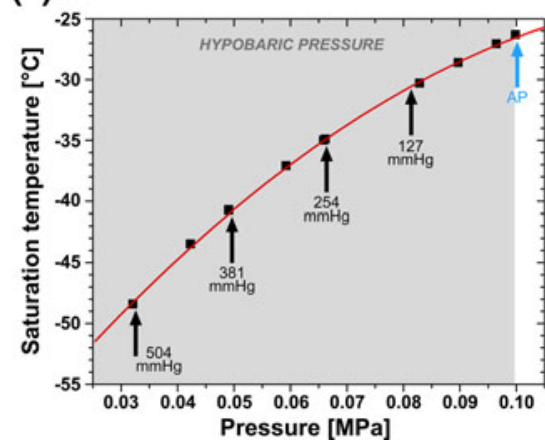

(b)

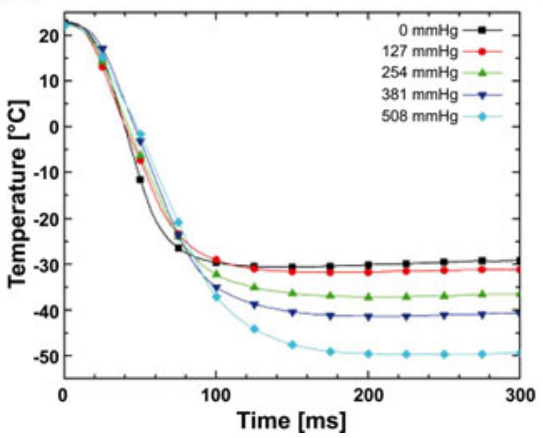

(c)

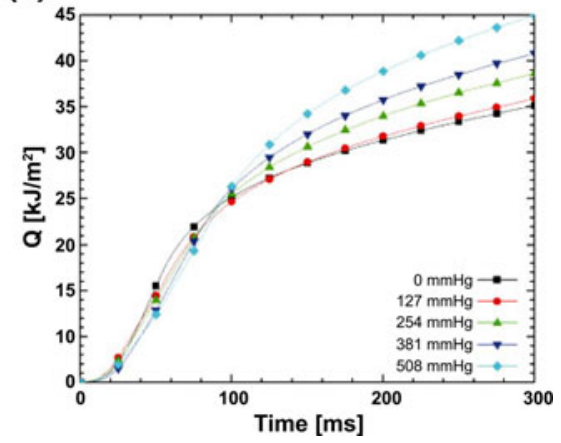

FIGURE 4. (a) Pressure-temperature (P-T) diagram for 1,1,1,2-tetrafluoroethane (R134a), showing reduced saturation (i.e., evaporation) temperatures with increasing hypobaric pressure (designated in $\mathrm{mmHg}$ and indicated in light gray). AP = atmospheric pressure. (b) Skin phantom surface temperatures as a function of time after initiation of a CSC spurt for 0 (AP), 127, 254, 381 , and $508 \mathrm{mmHg}$ of hypobaric pressure. (c) Skin phantom surface heat removal as a function of time for 0 (AP), 127, 254, 381, and $508 \mathrm{mmHg}$ of hypobaric pressure. $Q=$ total heat extraction. 

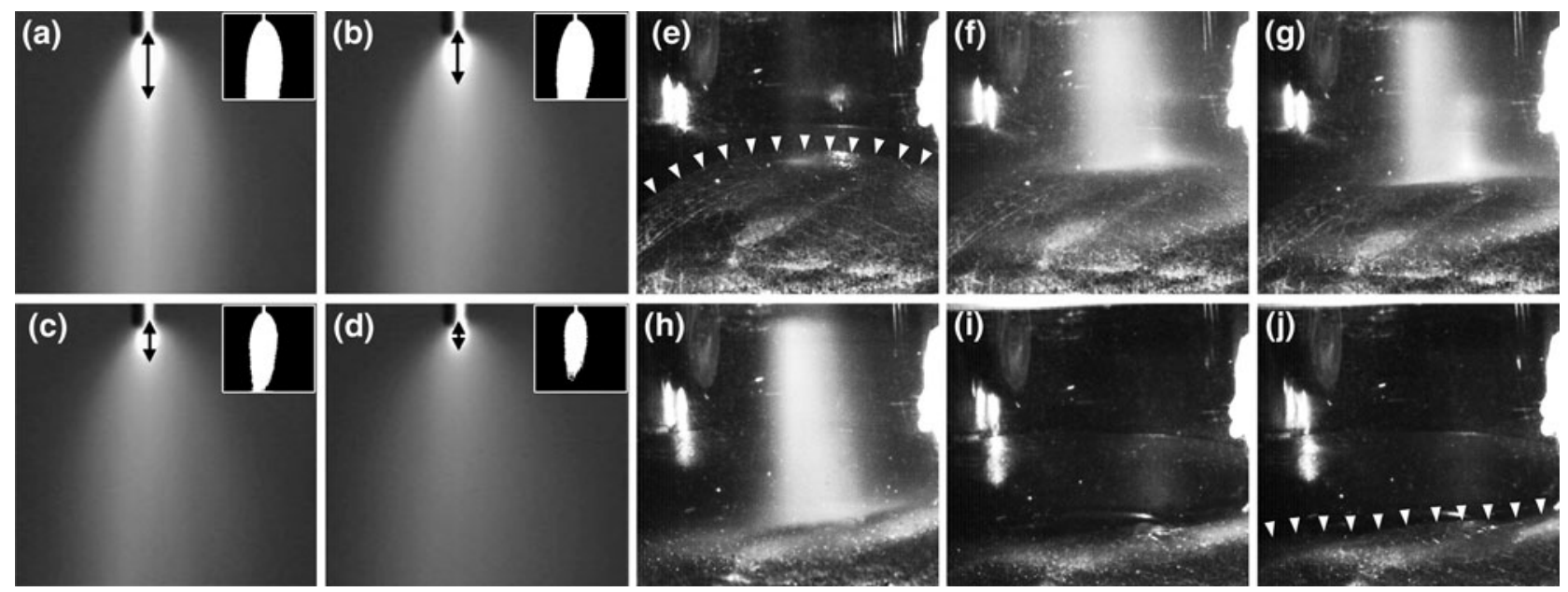

FIGURE 5. Isolated video frames of CSC spurts released within a vacuum chamber under atmospheric pressure (a, $760 \mathrm{mmHg})$ and at 254 (b), 381 (c), and $508 \mathrm{mmHg}$ (d) of hypobaric pressure. The frames were isolated at 100 ms into a 1-s CSC spurt. Arrows indicate approximate jet length based on the region of intense light reflection. The inserts are representations of droplet distribution for the respective CSC spurt. The images in panels (a-d) were converted to binary images and a grayscale intensity threshold cutoff of 85 was used to eliminate pixels with an intensity of $\mathbf{0 - 8 4}$. Accordingly, CSC spurts with a high droplet density (i.e., in a compact cloud) exhibit more reflection and thus a higher grayscale value. CSC spurts with a low droplet density (i.e., in a diffuse cloud) have a lower reflection and thus a lower grayscale value. The generated clouds in the inserts therefore provide an indication on the diffuseness or droplet distribution of a CSC spurt. Data modified from Aguilar et al. ${ }^{2}$ The next set of experiments (e-j) was performed on the skin of a healthy volunteer, showing frames from a CSC spray impingement video that corresponds to 0 (e), 32 (f), 64 (g), 100 (h), 128 (i), and $160 \mathrm{~ms}$ (j) during and after a 100-ms spurt at $254 \mathrm{mmHg}$ hypobaric pressure. The distance between the nozzle and the skin was $20 \mathrm{~mm}$. The arrowheads in (e) and (j) indicate the transition from a concave to flat skin surface before and after the CSC spurt, respectively. Data modified from Aguilar et al. ${ }^{2}$.

(concave) surface as deep as $2 \mathrm{~mm},{ }^{16}$ which decreases the maximum heat flux across the skin surface by as much as $30 \%$ relative to a flat surface. Contrastingly, local hypobaric pressure induces the formation of a convex surface (Fig. 5b), which abrogates the CSC spurt-induced indentation and minimizes cryogen "pooling" and thus decreases the adverse heat flux.

Finally, inasmuch as the air with a standard relative humidity is depleted at hypobaric pressures, the humidity within the chamber is significantly diminished. This effect in itself results in an enhancement of heat extraction from the skin, as documented by Torres et al.,${ }^{143}$ Majaron et al. ${ }^{93}$ and Ramirez-San-Juan et al. ${ }^{120}$ and prevents the build up of frost during a CSC spurt in the absence of a suction device because of the condensation and freezing of surface moisture on the skin. Frost formation and the evaporation of the cryogen pool are associated with reduced optical penetration depth ${ }^{43,120}$ and thus subtherapeutic photocoagulation. Moreover, frost formation may result in overcooling of the skin and corollary (epi)dermal injury. $38,77,119$

Overall, the combined effects of hypobaric pressure on CSC such as reduced spray temperature, better atomization and spray deposition, enhanced evaporation rate, stretched and convex skin surface, and lower local humidity result in a reduced skin surface temperature and an improvement in the overall heat extraction provided by CSC.

\section{COMBINED LASER TREATMENT AND OPTICAL IMAGING}

During the past three decades, experimental studies have focused on the development of methods to improve the efficacy of each PWS treatment session so as to reduce the total number of required sessions. Previous theoretical studies have established optimal laser parameters for treatment of model PWS blood vessels. However, PWS vascular anatomy and morphology is highly heterogeneous in a single lesion, as a result of which every singularly treated dermal volume essentially requires its unique set of laser parameters. Accordingly, the general consensus is that pretreatment knowledge of PWS skin characteristics could be used to guide laser parameter selection on a personalized basis ${ }^{148}$ so as to treat the entire PWS more effectively.

Considerable research effort has been invested in the noninvasive characterization of PWS skin regions and the quantification of skin characteristics such as blood vessel diameter and depth. ${ }^{31,94,95,100,104,151,156}$ Currently, the novel instrumentation under development is limited due to the inherent complexity of PWS microvascular architecture ${ }^{126,132}$ and the lack of knowledge on how exactly the extracted skin characteristics can be used to guide treatment parameter selection.

In an effort to establish a relationship between diagnostic/prognostic optical techniques and laser- 
tissue interactions, we have employed the rodent dorsal window-chamber model as an in vivo platform ${ }^{19,25,30,76}$ to evaluate phototherapies designed to eliminate aberrant blood vessels. Based on data collected from over 300 experiments, it was shown that, in the absence of complete acute photocoagulation of the irradiated vessels, the laser-irradiated region of interest (ROI) remains perfused. These findings provided compelling evidence for the supposition that complete acute photocoagulation of PWS vessels to a certain dermal depth may be important to achieve optimal blanching, as has been addressed earlier in clinical studies. ${ }^{48,72}$

Purpura formation (local hemorrhage due to laserinduced vascular damage) is typically used as a qualitative clinical endpoint and prognostic indicator for photocoagulation. However, the extent of purpura formation is not an unequivocally accurate indicator of treatment outcome. In an exemplary computational modeling study, Pfefer et al. ${ }^{114}$ demonstrated that incomplete thermal damage to superficial PWS vessels readily occurs because of a shielding phenomenon. Specifically, the presence of PWS vessels proximal to the skin surface will reduce the optical fluence available in any vessels directly below, thus reducing the probability of complete vessel photocoagulation. In this case, purpura formation due to photocoagulation of the proximal vessels is expected to occur, but the degree of lesion blanching is expected to be compromised because of incomplete photocoagulation of the optically shielded vessels. Furthermore, in our clinical practice, we have observed significant lesional blanching at minimal purpura formation.

Based on the hypothesis that complete acute photocoagulation must be achieved and the problems with using the purpura threshold as a metric of photocoagulation, a clinic-friendly laser speckle imaging (LSI) system was developed ${ }^{25,30,76}$ to assess the degree of photocoagulation achieved during laser surgery. This method would enable clinicians to visualize the degree of skin perfusion after laser therapy as a means to gauge the necessity of immediate retreatment of specific regions in which perfusion had persisted.

The LSI methodology has been described by Boas and Dunn. ${ }^{23}$ In summary, coherent light from typically $633-\mathrm{nm} \mathrm{HeNe}$ or $785-\mathrm{nm}$ diode lasers is used to illuminate a ROI. A CCD or CMOS camera is used to image the illuminated ROI, whereby a speckle pattern is observed. Local fluctuations in the speckle pattern occur because of motion of optical scatterers such as erythrocytes. When the image exposure time is greater than a characteristic fluctuation frequency of the scatterers, local blurring of the speckle pattern occurs. Subsequently, a local speckle contrast value $(K)$ is calculated for each pixel using various algorithms. ${ }^{28,42,118,121} K$ is high when the degree of local blurring is low (i.e., stationary scatterers), and $K$ is low when the degree of local blurring is high (i.e., moving scatterers) (Fig. 6). Based on the assumptions made about the motion characteristics (e.g., Brownian, ordered) of the moving scatterers, estimates of the speckle decorrelation time $\left(\tau_{\mathrm{c}}\right)$ are made from the $K$ value. The relative degree of scatterer motion, which is referred to as the speckle flow index (SFI), is assumed to be the reciprocal of $\tau_{\mathrm{c}}$.

A primary design consideration during the development of the LSI instrument was the ability to collect raw speckle images with the patient positioned as comfortably as possible. To this end, an articulated arm was used as the base platform to provide flexibility in instrument positioning. ${ }^{73}$ Continuous wave light emitted from a 633-nm HeNe laser was delivered to the ROI with an optical fiber, and a diffusing glass substrate attached to the fiber output was used to homogenize the emitted beam. Raw speckle reflectance images were collected with a 12-bit, thermoelectrically cooled CCD camera (2000R, QImaging, Burnaby,
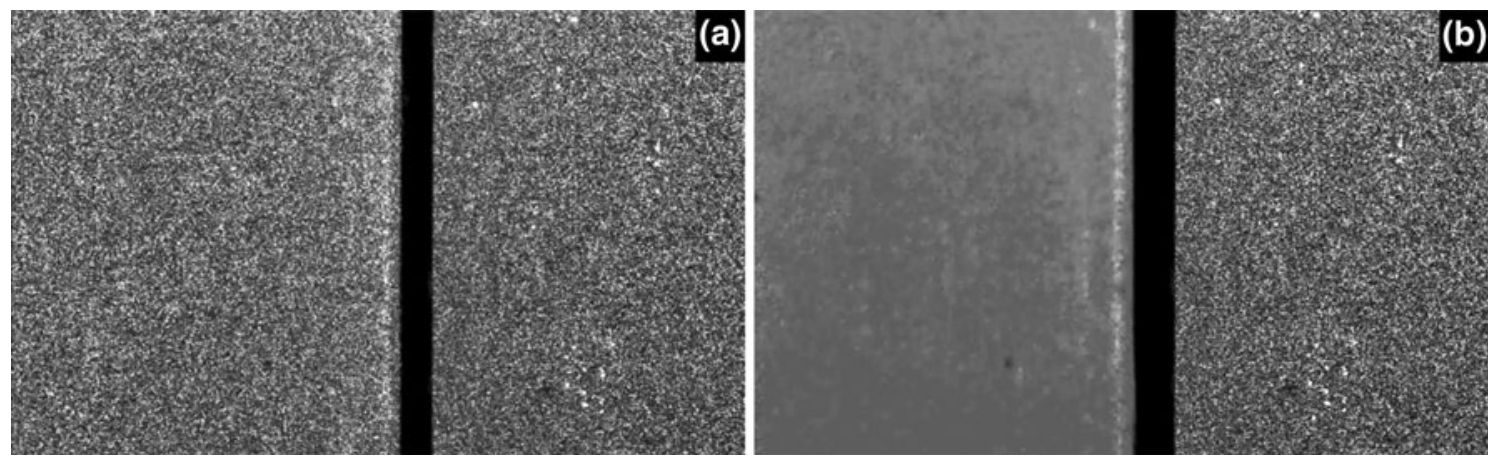

FIGURE 6. The local speckle contrast changes due to scatterer motion. A 633-nm HeNe laser was used to irradiate two identical white silicone blocks (the black vertical rectangle is the gap between the two blocks). (a) When both blocks are stationary, a speckle pattern is visualized for both blocks. (b) When the left block is manually moved, the speckles become blurred, which concurs with a reduction in speckle contrast $(K)$. The lower the value of $K$, the higher the degree of scatterer motion (i.e., flow when employed on the skin, where erythrocytes comprise the scatterers). 


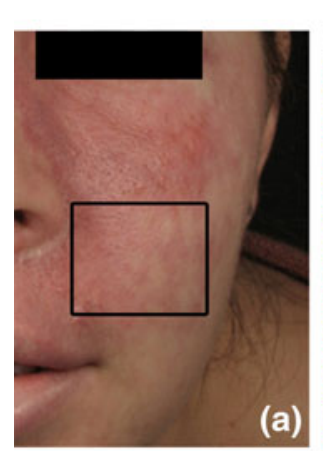

\section{LSI before laser irradiation}

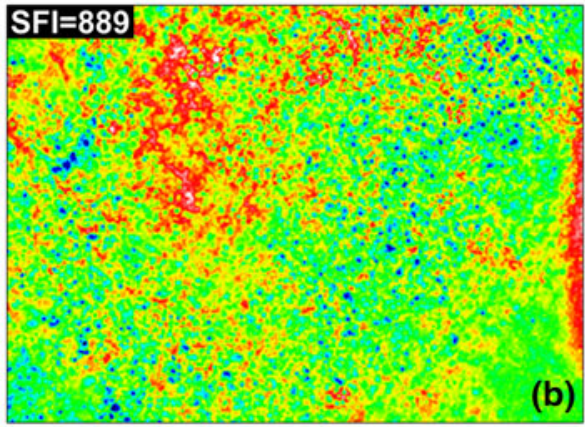

NO FLOW
LSI after laser irradiation

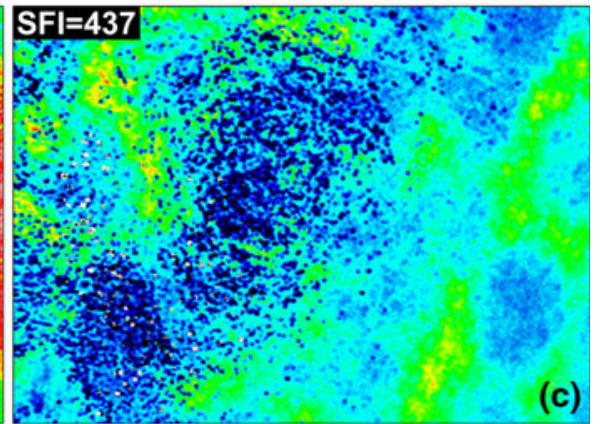

1546 HIGH FLOW

FIGURE 7. (a) Cross-polarized color image of a patient with a faint PWS. The black rectangle delineates the region imaged with LSI. SFI maps, which indicate relative flow velocities according to the indexed color (scale bar), were collected before (b) and $40 \mathrm{~min}$ after completion of laser treatment with a 595-nm pulsed dye laser. Some of the regions that had been irradiated exhibited persistent perfusion, indicating incomplete photocoagulation of the PWS vasculature. Images adapted from Huang et al. ${ }^{73}$.

(a)

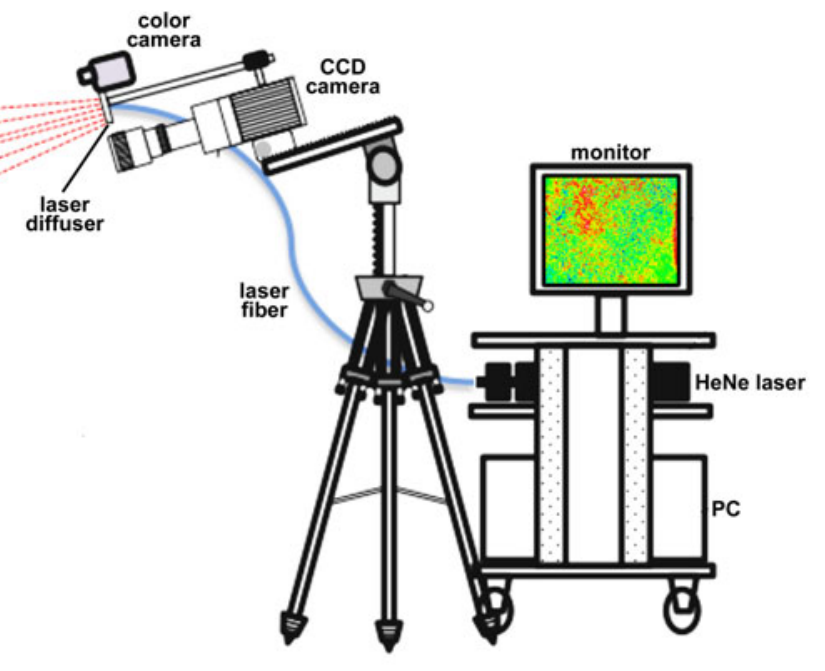

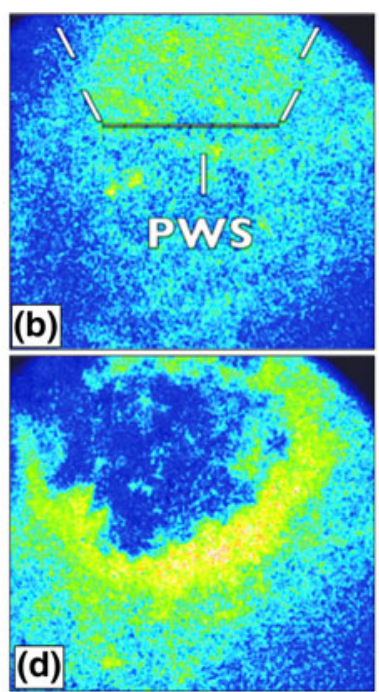

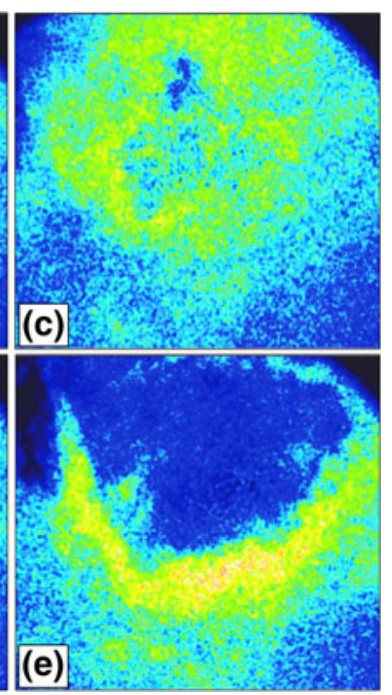

FIGURE 8. (a) Schematic representation of the second-generation LSI instrument currently in use in the operating room during laser surgery of PWSs. SFI images were extracted from a real-time video feed taken before (b) and at various stages during laser surgery of a PWS: (c) after completion of alexandrite laser therapy, (d) after one pass of a 595-nm pulsed dye laser, (e) after a subsequent pass of the 595-nm pulsed dye laser.

Canada) with a sensor size of $1600 \times 1200(\mathrm{H} \times \mathrm{W})$ pixels. Image data were transferred to a PC for storage, processing, and visualization. Custom-written LabVIEW software was used to control all aspects of image acquisition and processing. With a macro lens attached to the camera, the size of the ROI was $4 \times 3 \mathrm{~cm}$

The initial data collection focused on LSI performed outside of the operating room. ${ }^{73,74}$ Raw speckle reflectance image sequences were collected before and $\sim 40$ min after treatment was completed. Based on a pilot study involving measurements from 76 sites on 20 subjects, we observed that a decrease in skin perfusion occurred at a lower-than-expected rate $(72 \%)$, and that regions of persistent perfusion were frequently present
(44\%) (Fig. 7), which underscored the need for objective blood flow assessment during laser surgery of PWSs.

Next, we developed a second-generation, clinicfriendly LSI instrument encompassing the following engineering aims: (1) blood flow images should be displayed in real time and (2) the instrument should operate without perturbing the standard course of laser treatment. To achieve aim 1 , we designed software that enabled speckle contrast and SFI calculations to be performed on a graphics processing unit (GPU) card, ${ }^{162}$ which enabled real-time LSI of blood flow. To achieve aim 2, we developed a tripod-based LSI instrument that is easy to position before laser treatment and easy to reposition during surgery (Fig. 8a). 
With the use of this instrument, the clinician has immediate visual feedback on the degree of photocoagulation achieved at any perioperative time point. A case study is presented in Figs. 8b-8e for illustrative purposes. A patient with a PWS on the left side of her face was treated initially with a $755-\mathrm{nm}$ alexandrite laser, followed by treatment with a $595-\mathrm{nm}$ pulsed dye laser. After treatment with the alexandrite laser, a slight reduction in blood flow was observed, with a surrounding region of hyperemia. A hyperemic region is frequently observed and is attributed to compensatory vasodilation of the intact vasculature in part due to thermoregulation. Following treatment with the pulsed dye laser, a marked reduction in blood flow was observed, with some regions of persistent perfusion. A subsequent pass with the pulsed dye laser resulted in homogeneous shutdown of blood flow in the treated region. A clinical study is currently underway to determine whether immediate retreatment on the basis of LSI profiles leads to a significant improvement in treatment outcome.

\section{SITE-SPECIFIC PHARMACO-LASER THERAPY}

\section{Endovascular Laser-Tissue Interactions in Incompletely Photocoagulated Vessels}

The concept of SP was developed around the photothermal response, namely, the formation of a thermal coagulum due to supracritical heat generation in the vascular lumen with laser light. Complete photocoagulation of blood vessels is exclusively associated with the photothermal component of endovascular laser-tissue interactions, i.e., photocoagulation. However, in incompletely photocoagulated blood vessels, i.e., blood vessels in refractory PWSs, the photothermal

\section{PHOTOTHERMAL RESPONSE}
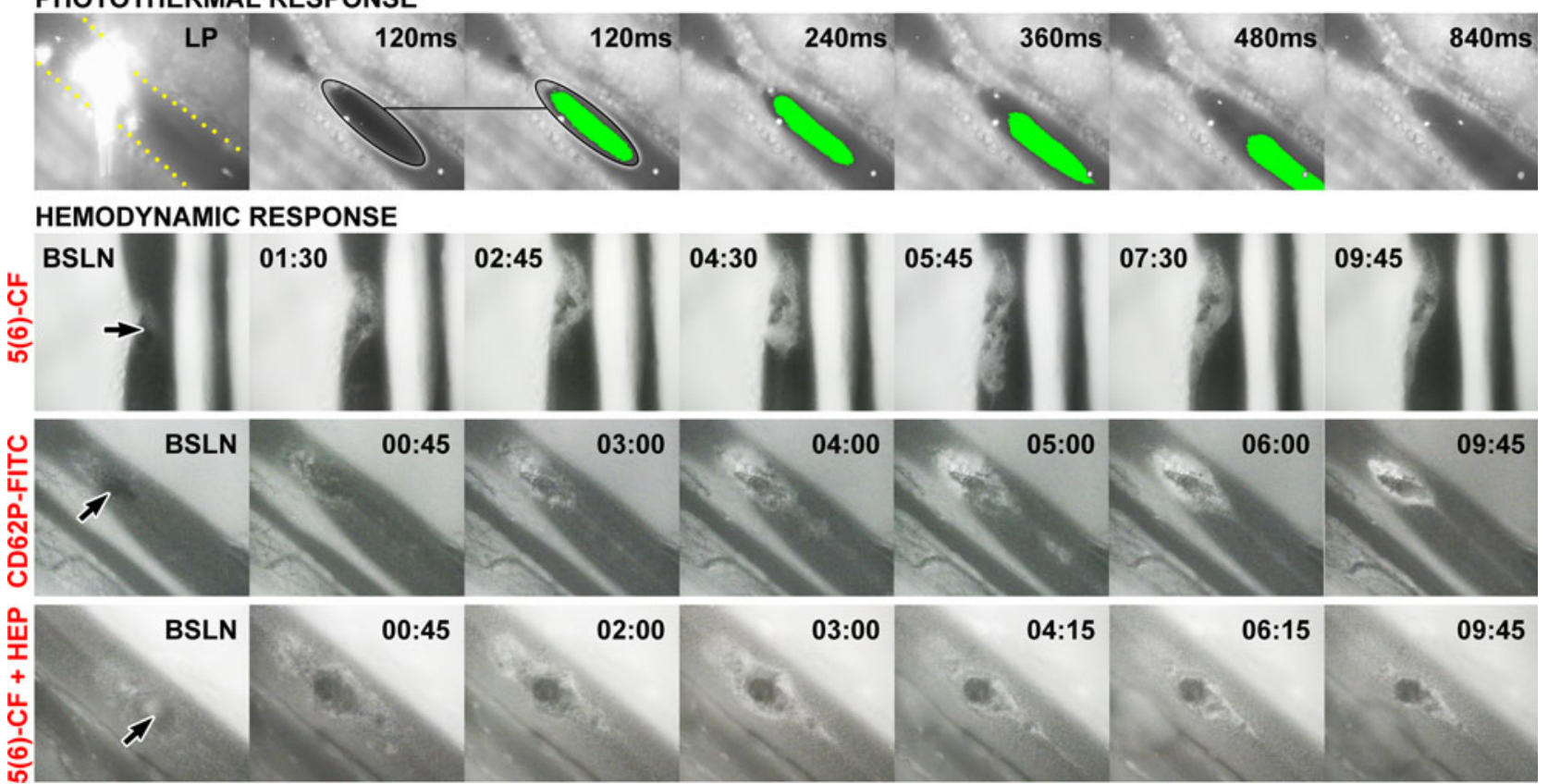

FIGURE 9. The photothermal and hemodynamic responses studied in hamster dorsal skin fold venules. ${ }^{19,68}$ The venules, delineated by yellow dots in the upper left panel, were irradiated with a frequency-doubled Nd:YAG laser (532 nm, radiant exposure of $289 \mathrm{~J} / \mathrm{cm}^{2}, 30-\mathrm{ms}$ pulse duration, and a spot size of $2.3 \times 10^{-3} \mathrm{~mm}^{2}$ ) to generate subocclusive thermal coagula. Endovascular events were imaged by intravital fluorescence microscopy in combination with brightfield microscopy (photothermal response only). The photothermal response (top row) encompassed the formation of a thermal coagulum (encircled) that remained attached (not shown) or dislodged at the site of laser irradiation. The thermal coagulum was imaged in time, indicated in the upper right corner. Thermal coagula were given a pseudocolor from the third panel onward for better visualization, obtained by intensity thresholding. The second and third panels from the left are identical to demonstrate the accuracy with which the thermal coagula were contoured. Fluorescent microspheres (bright dots in the venule) were infused to monitor blood flow. Venular occlusion was never achieved at these laser parameters, corresponding to the endovascular damage profile in refractory PWS vessels. The hemodynamic response was studied following fluorescent labeling of platelets with $5(6)$-carboxyfluorescein (5(6)-CF) ${ }^{69}$ in vivo and after laser irradiation (baseline, BSLN). The time after laser irradiation is provided in the upper left or right corner (min:s), and the thermal coagulum is indicated by the arrow. Thrombosis, which encompassed platelet aggregation at and around the thermal coagulum, was characterized by a growth phase (BSLN-06:15) and a subsequent deterioration phase (06:15-15:00). It was further shown that the aggregating platelets become activated, as evidenced by the positive staining with fluorescently labeled antiCD62P (P-selectin) antibodies, an activation-dependent epitope on platelets and endothelial cells ("CD62P-FITC" row). Moreover, the involvement of the coagulation cascade was confirmed in experiments in which platelets were stained with $5(6)$-CF and heparin, an inhibitor of coagulation, was co-infused ("5(6)-CF + HEP" row). The presence of heparin reduced the mean maximum lesional size by $51 \%$ and significantly reduced the duration of the thrombus growth phase. 
response triggers an additional effect, namely a hemodynamic response (thrombosis). ${ }^{64,68,140}$ The hemodynamic response requires residual blood flow for the supply of platelets and coagulation factors, which is absent in completely photocoagulated vasculature. Recent in vivo studies have demonstrated that the hemodynamic response entails both primary (platelet adhesion, activation, and aggregation) and secondary hemostasis (coagulation), and that the hemodynamic response manifests itself even in the absence of a thermal coagulum. The photothermal and hemodynamic responses are summarized in Fig. $9^{19,68,69}$ and lie at the basis of site-specific pharmaco-laser therapy (SSPLT). thrombosis constitutes an inherent component of endovascular laser-tissue interactions in incompletely coagulated vessels. With complete vascular occlusion as the primary goal of SP, the hemodynamic response may be used as a means to occlude vascular lumens that have not been completely thermolysed during SP. In this respect, manipulating the hemodynamic response by the administration of prothrombotic and/ or antifibrinolytic pharmaceuticals may function as an adjuvant in complementing an inadequate endovascular damage profile in refractory PWSs. ${ }^{64,65,68}$ The envisaged pharmacodynamics of SSPLT are illustrated in Fig. 10 in the context of the photothermal and hemodynamic response.

\section{Principles of Site-Specific Pharmaco-Laser Therapy}

SSPLT is a development stage treatment modality that combines conventional laser therapy, SP, with the prior systemic administration of prothrombotic and/or antifibrinolytic pharmaceutical agents encapsulated in a targeted drug delivery system (DDS). The underlying principles of SSPLT are based on the finding that

\section{Drug Delivery Platform for Site-Specific Pharmaco-Laser Therapy}

The potential hazard of parentally administering prothrombotic and/or antifibrinolytic substances to non-coagulopathic patients is impairment of the

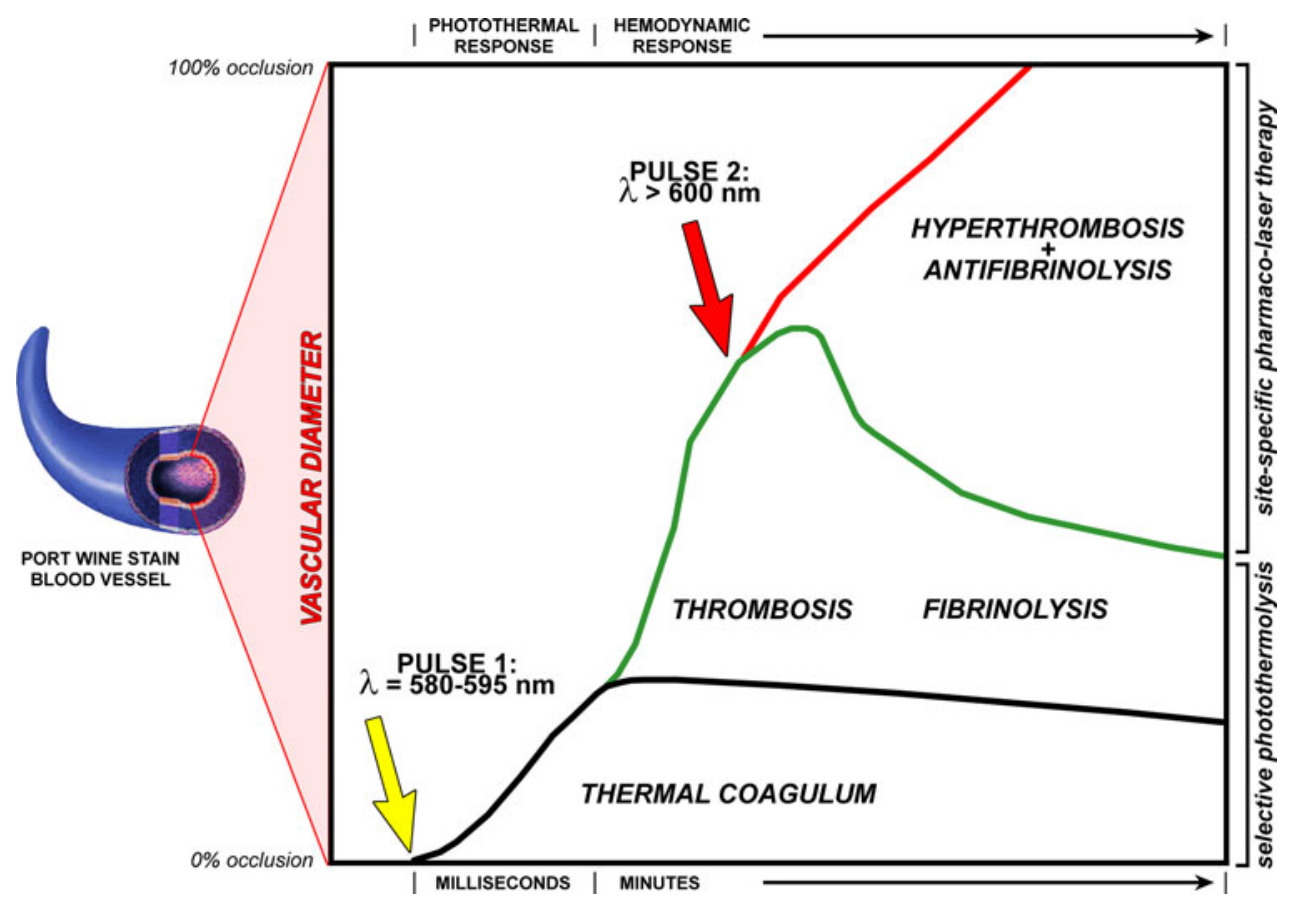

FIGURE 10. Mechanistic illustration of the envisaged treatment modality. A PWS blood vessel is depicted on the left and the $y$ axis represents its vascular diameter. The bottom of the $y$-axis indicates $0 \%$ occlusion, the top of the $y$-axis represents $100 \%$ occlusion. The $x$-axis represents time, which is divided in a photothermal response time frame (i.e., laser irradiation) and a hemodynamic response time frame (thrombosis and fibrinolysis). When a patient with a recalcitrant PWS is treated with a laser (pulse 1, yellow arrow), the blood vessel is only partially occluded by photocoagulated blood (thermal coagulum, black line). The laser irradiation triggers thrombosis (green upward line), which extends the degree of vascular occlusion, but not to the level of complete occlusion. Moreover, in time the thrombus breaks down because of enzymatic deterioration (fibrinolysis, green downward line). In case of site-specific pharmaco-laser therapy, photoactivatable nanoparticulate drug delivery carriers are infused into the patient that contain pharmaceutical agents to promote thrombus growth and inhibit thrombus breakdown. Once these drug carriers have accumulated in the thrombus after the first laser pulse, a second laser pulse (pulse 2, red arrow) is used to induce drug release and subsequent pharmacological augmentation of thrombosis and deterrence of fibrinolysis (hyperthrombosis + antifibrinolysis, green line). The extent of hyperthrombosis and antifibrinolysis is exacerbated to such a degree that the entire blood vessel becomes occluded, marking the therapeutic endpoint (i.e., good clearance). 
(a)

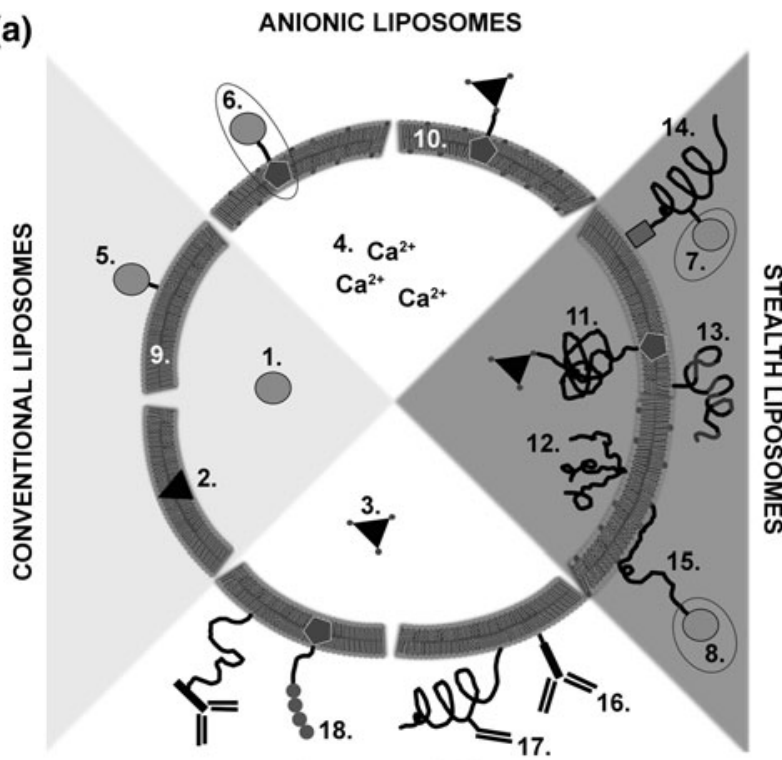

TARGETED LIPOSOMES

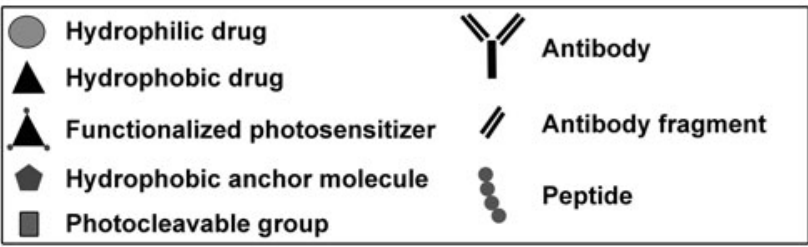

(b)

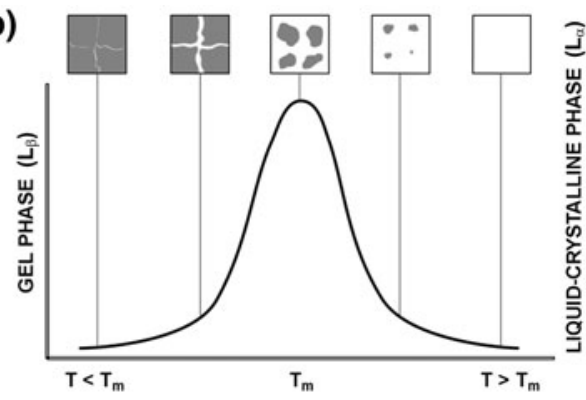

(c)
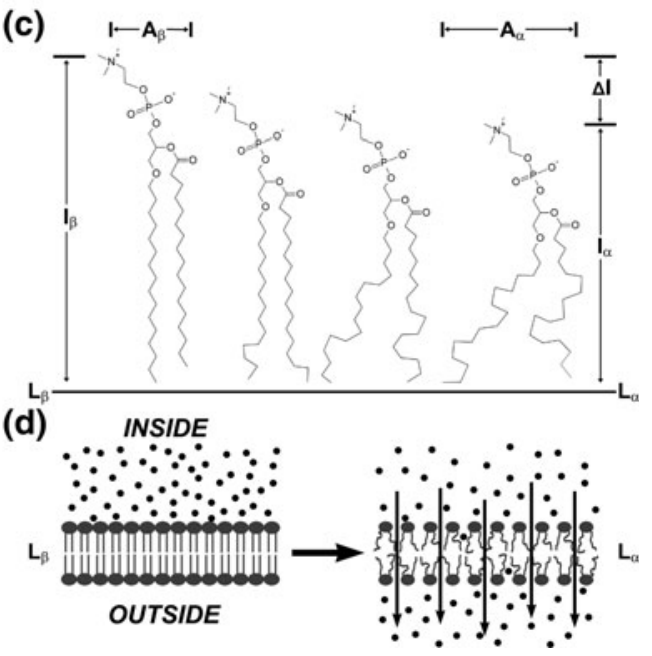

FIGURE 11. (a) Generic scheme of possible liposomal formulations for site-specific pharmaco-laser therapy. Liposomes are nanoscopic fat droplets that consist of a bilayer of phospholipids and an inner aqueous compartment. The possible liposomal formulations can be divided into four main categories: conventional liposomes, anionic liposomes, sterically stabilized liposomes, and targeted liposomes. Each main category may encompass any of the following subcategories: (I) types of drugs: 1. hydrophilic drugs (e.g., tranexamic acid); 2. hydrophobic drugs (e.g., photosensitizers); 3. functionalized hydrophobic drugs (e.g., functionalized photosensitizers); 4. ions (e.g., calcium); (II) drug grafting methods: 5. (covalent) attachment to a component (phospho)lipid; 6. (covalent) attachment to an anchor molecule (e.g., cholesterol); 7. (covalent) attachment to a polymer side chain (e.g., polyethylene glycol, PEG); 8. (covalent) attachment to a functionalized distal end of a polymer; (III) membrane composition: 9. phosphatidylcholines; 10. phosphatidylcholines with a molar fraction of anionic/cationic (phospho)lipids; (IV) methods of steric stabilization: 11. single chain polymer (e.g., polyethylene glycol, PEG); 12. multichain polymer; 13. multiblock copolymer (e.g., dior triblock copolymers); 14. photocleavable polymers (e.g., PEGylated plasmalogens); 15. adsorbable polymers (onto anionic/ cationic membrane surface); (V) methods of targeting: 16. antibodies; 17. antibody fragments (e.g., Fab' fragments); and 18. peptides. The main categories are not mutually exclusive; e.g., sterically stabilized liposomes may contain anionic membrane constituents as well as antibodies for targeting. (b) The gel-to-liquid crystalline phase transition of a lipid bilayer. Lipid bilayers principally exist in a gel phase $\left(L_{\beta}\right)$ at temperatures $(T)$ below phase transition temperature $\left(T_{\mathrm{m}}\right)$ and in a liquid crystalline phase $\left(L_{\alpha}\right)$ at $T>T_{m}$, depicted on the $x$-axis. Lipid packing is highly ordered in $L_{\beta}$ and somewhat disordered in $L_{\alpha}$, accounting for the high and lower degree of membrane impermeability, respectively. The maximum in several physical properties and characteristics, such as (all on $y$-axis) specific heat, membrane permeability, and interphase boundary (between the melted liquid phase and still solid gel phase) are all at a maximum at $T_{\mathrm{m}}$, and define $T_{\mathrm{m}}$. During heating, grain boundaries arise in the bilayer that demarcate lipid domains in $L_{\beta}$ and $L_{\alpha}$ (gray and white areas in top panels, respectively). The $L_{\beta}-L_{\alpha}$ interface is characterized by lipid packing defects that impose significant membrane permeability during which the release of liposome-encapsulated molecules becomes possible. Figure partially adapted from Needham et al. ${ }^{103}$ (c) Diagram of dipalmitoyl phosphocholine (16:0) in $L_{\beta}$ (left molecule) transiting to $L_{\alpha}$ (right molecule). The horizontal solid line marks the midplane of the bilayer. The figure is a superimposable representation at the molecular level of the thermogram/phase diagram in (b). The hydrocarbon chains of the $L_{\beta}$ phospholipid are in an all-trans state before $T_{\mathrm{m}}$. Following phase transition into $L_{\alpha}$, the hydrocarbon chains exhibit considerable rotameric disorder that is characteristic of linear alkyl chains at $T>T_{\mathrm{m}}$. The thinning of the membrane is shown by $\Delta I=I_{\mathrm{b}}-I_{\mathrm{a}}$, and the molecular areas $A_{\beta}$ (gel phase) and $A_{\alpha}$ (liquid crystalline phase) are indicated, all contributing to the increased permeability of the bilayer and corollary release (d, arrows) of encapsulated molecules (d, black spheres). (c) Adapted from Nagle JF. ${ }^{102}$.

hemostatic "checks and balance" system. Consequently, the pharmaceutical efficacy of the prothrombotic and antifibrinolytic agents must be constrained to the laser-treated birthmark region only so that the regulation of naturally occurring hemostatic events is not compromised. ${ }^{64}$ This can be achieved by encapsulating the pharmaceutical agents into a DDS.

\section{Liposomes as Drug Delivery System for Site-Specific Pharmaco-Laser Therapy}

For SSPLT, liposomes (nanoscopic fat droplets) constitute the most advantageous carrier system because of their manipulatable attributes and their ability to encapsulate hydrophilic and lipophilic molecules at 
high efficiencies (Fig. $11 \mathrm{a}^{87}$ ). In addition to the inherent non-to-very-low toxicity of neutral phospholipids, ${ }^{26,98}$ liposomes can be modified compositionally to facilitate the unique prerequisites of the DDS. Enhancement of the in vivo circulation time can be accomplished by proper sizing ${ }^{13}$ and by the conjugation of polyethylene glycol (PEG) to polar phospholipids, usually phosphatidylethanolamine. ${ }^{4,81,112,127}$ Inclusion of PEG chains is also useful for designing immunotargeting liposomes capable of homing to the target site through the attachment of antibodies or Fab' fragments to a chemically modified distal end of a liposome-grafted PEG chain. ${ }^{62,129,141,142}$ Antibodies directed against platelet epitopes such as P-selectin, which was shown to be expressed but not to play a role in laser-induced thrombosis, ${ }^{68}$ or antibodies against fibrin are suitable candidates for SSPLT of PWSs. (a)

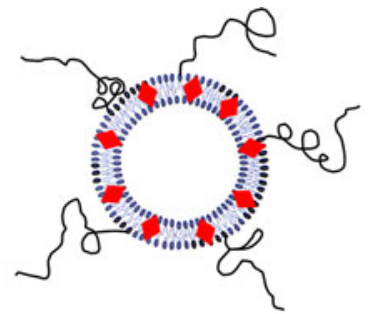

(d)

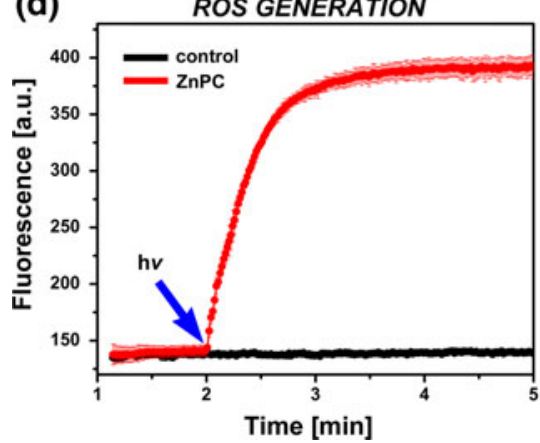

(b)

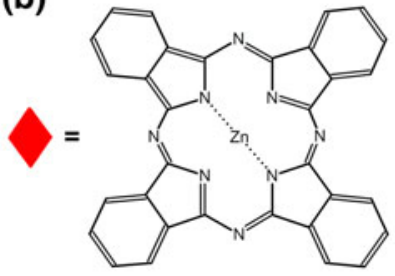

(e) BIOMEMBRANE PERMEABLIZATION

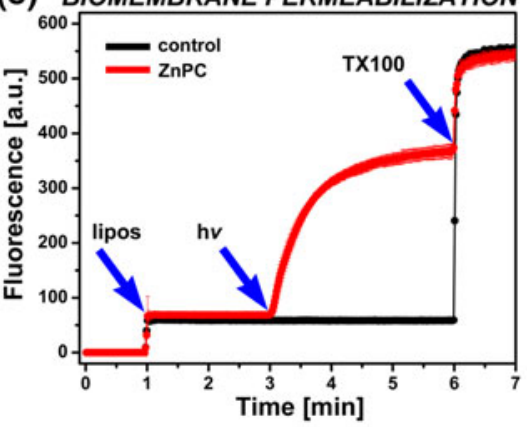

(c)

OXIDATION OF BIOMOLECULES
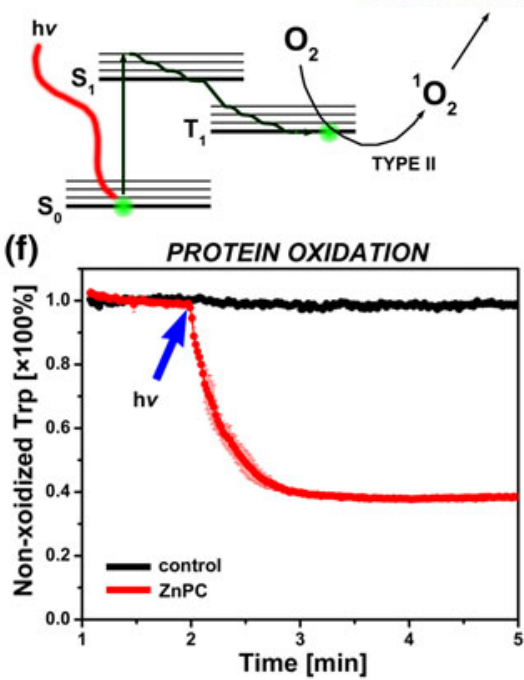

FIGURE 12. (a) Model liposomal DDS for prothrombotic site-specific pharmaco-laser therapy, consisting of phosphatidylcholine lipids, cholesterol, and a molar fraction of phosphatidylethanolamine-conjugated PEG. The PEG can be used to conjugate antibodies for immunotargeting to the laser-induced thrombus via e.g., P-selectin or fibrin. The liposomes encapsulate a second generation photosensitizer, zinc phthalocyanine (ZnPC, red diamonds), in the lipid bilayer. The chemical structure of ZnPC is provided in (b). (c) ZnPC is activated by irradiation with resonant light (absorption maximum at $674 \mathrm{~nm}$ ), causing electrons to transit from the ground $\left(S_{0}\right)$ to the first excited state $\left(S_{1}\right)$ and subsequently to the triplet state $\left(T_{1}\right)$. The photodynamic effect proceeds from the $\mathrm{T}_{1}$ state via energy transfer (type II) to molecular oxygen, yielding the highly cytotoxic and thrombogenic singlet oxygen $\left({ }^{1} \mathrm{O}_{2}\right)$, a ROS. The generation of ROS is shown in (d), where ZnPC-encapsulating liposomes composed of 1,2-dipalmitoyl-sn-glycero-3phosphocholine (DPPC, $66 \mathrm{~mol} \%$ ), cholesterol (30 mol\%), and 1,2-distearoyl-sn-glycero-3-phosphoethanolamine-PEG (DSPE-PEG, $4 \mathrm{~mol} \%$ ) were suspended in buffer containing protonated dichlorofluorescein $\left(\mathrm{DCFH}_{2}\right)$, a probe that becomes highly fluorescent upon oxidation $\left(\mathrm{DCFH}_{2} \rightarrow \mathrm{DCF}, \lambda_{\mathrm{ex}}=500 \pm 5 \mathrm{~nm}, \lambda_{\mathrm{em}}=522 \pm 5 \mathrm{~nm}\right.$ ). The liposome suspension was irradiated with a $670-\mathrm{nm}$ solid-state diode laser ( $\mathrm{h} v$, blue arrow) to activate $\mathrm{ZnPC}$. The formation of DCF was monitored by fluorescence spectroscopy in timebased acquisition mode. Oxidation of $\mathrm{DCFH}_{2}$ occurred immediately upon laser irradiation of ZnPC-liposomes (red trace, ZnPC:lipid ratio of 0.004), as evidenced by the increase in DCF fluorescence at $522 \mathrm{~nm}$. Irradiation of ZnPC-lacking liposomes (control, black trace) did not result in the generation of ROS and thus DCF fluorescence. In (e), a similar setup was used as in (d) to demonstrate ROS-mediated membrane disruption in cell phantoms composed of DPPC (39 mol\%), distearoylphosphocholine (10 mol\%), cholesterol (20 mol\%), dipalmitoylphosphoserine (20 mol\%), dioleoylphosphocholine (5 mol\%), 1-stearoyl-2-docosahexaenoylphosphocholine (5 mol\%), and $1 \mathrm{~mol} \% \alpha$-tocopherol (5 mM final lipid concentration). The ZnPC liposomes encapsulated calcein at a self-quenching concentration $\left(53 \mathrm{mM}, \lambda_{\mathrm{ex}}=488 \pm 5 \mathrm{~nm}, \lambda_{\mathrm{em}}=522 \pm 5 \mathrm{~nm}\right)$ in the aqueous compartment. Disruption of the liposomal membrane results in leakage of calcein from the liposomes, abrogation of fluorescence quenching, and increase in calcein fluorescence. At $t=1 \mathrm{~min}$, liposomes (lipos, blue arrow) were added to the cuvette in the fluorescence spectrometer, at $t=3 \mathrm{~min}$ the suspension was irradiated with a $670-\mathrm{nm}$ laser $(\mathrm{h} v$, blue arrow), and at $t=6 \mathrm{~min}$ Triton X-100, a detergent that solubilizes the liposome membrane, was added (TX100, blue arrow) to determine complete calcein release from the liposomes. Irradiation of ZnPCencapsulating liposomes (red trace) resulted in rapid leakage of calcein from the liposomes, probably due to oxidative modification of phospholipids, whereas irradiation of ZnPC-lacking liposomes had no effect on membrane permeabilization. In (f) it is shown that the ROS produced by ZnPC liposomes have the ability to oxidize amino acid residues in bovine serum albumin (BSA). A similar experiment was performed as in (d), only the ZnPC-liposomes were suspended in buffer containing BSA. The fluorescence of tryptophan $\left(\lambda_{\mathrm{ex}}=280 \pm 5 \mathrm{~nm}, \lambda_{\mathrm{em}}=360 \pm 5 \mathrm{~nm}\right)$, an autofluorescent amino acid in BSA, was measured as a function of time. Oxidation of tryptophan leads to abrogation of fluorescence. At $t=2 \mathrm{~min}$, the liposome-containing cuvette was irradiated with 670$\mathrm{nm}$ light (hv, blue arrow). Oxidation of tryptophan occurred in samples containing ZnPC-liposomes (red trace) but not in samples containing ZnPC-lacking liposomes (black trace), as evidenced by the rapid reduction in fluorescence intensity. Data were normalized to tryptophan fluorescence at $t=1.99 \mathrm{~min}$. The data in panels $(\mathrm{e}, \mathrm{f})$ comprise mean $\pm \mathrm{SD}$ traces from $n=3$ experiments (ZnPC-liposomes) and single traces for control liposomes. All data is unpublished. 
An additional benefit of liposomal DDSs is that the drug release mechanism can be accommodative to the specific application and physiological context. Active triggering mechanisms can be engineered around thermosensitivity (membrane destabilization through kinetic energy) ${ }^{10,103}$ (Figs. 11b-11d $\mathrm{d}^{102,103}$ ), photochemical modification of bilayer structure, ${ }^{54,131}$ or a combination thereof. Hyperthermia has been employed in numerous liposomal formulations in vitro and in vivo $^{10,17,50,51,82,97,99,103,158}$ to initiate a thermotropic alteration in membrane permeability that will lead to a rapid, triggered release of the loaded molecules.

Different liposomal formulations will be employed for the prothrombotic and the antifibrinolytic SSPLT modalities. Principally, both liposomal formulations consist of dipalmitoyl phosphocholine (DPPC), contain a $4 \%$ molar fraction of PEG-conjugated distearoyl phopshoethanolamine (DSPE-PEG), and are targeted

(a)

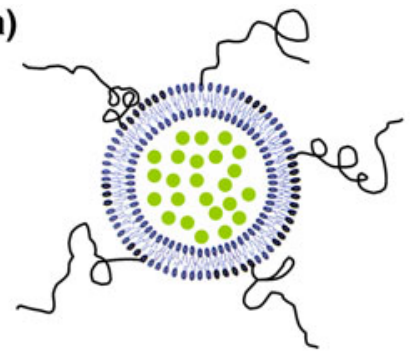

(d)

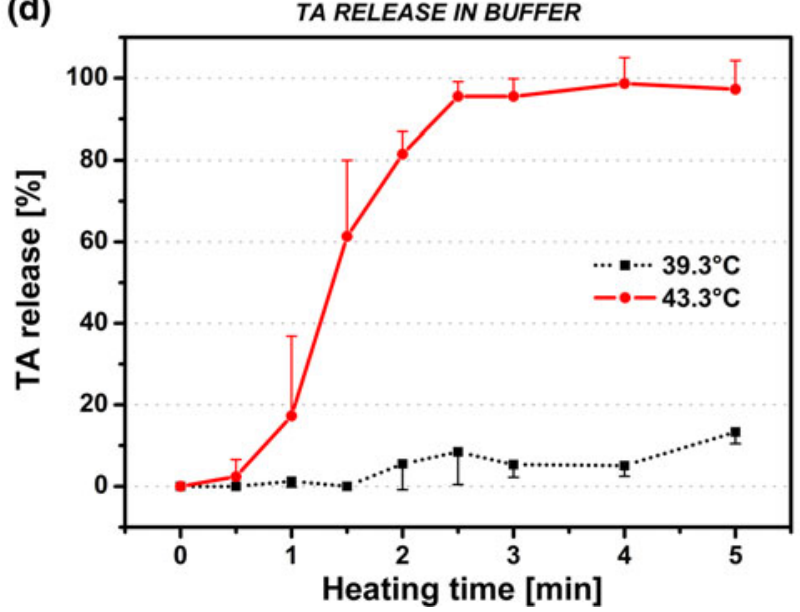

to thrombi in partially occluded PWS vasculature through. e.g., the conjugation of thrombus-specific antibodies. However, liposomes for prothrombotic SSPLT encapsulate a photosensitizer in the lipid bilayer that amplifies the hemodynamic response by reactive oxygen species (ROS) following activation of the photosensitizer by resonant light. Contrastingly, liposomes for antifibrinolytic SSPLT are thermosensitive and contain an antifibrinolytic agent in the aqueous compartment that is actively released upon a heat stimulus.

\section{Liposomes for Prothrombotic Site-Specific Pharmaco- Laser Therapy}

Rather than using classical prothrombotic agents, which typically are expensive and heat-labile proteins, the prototype liposomal DDS for prothrombotic SSPLT contains zinc phthalocyanine (ZnPC), a second

\section{(c)}

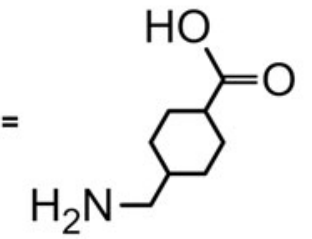

(e)
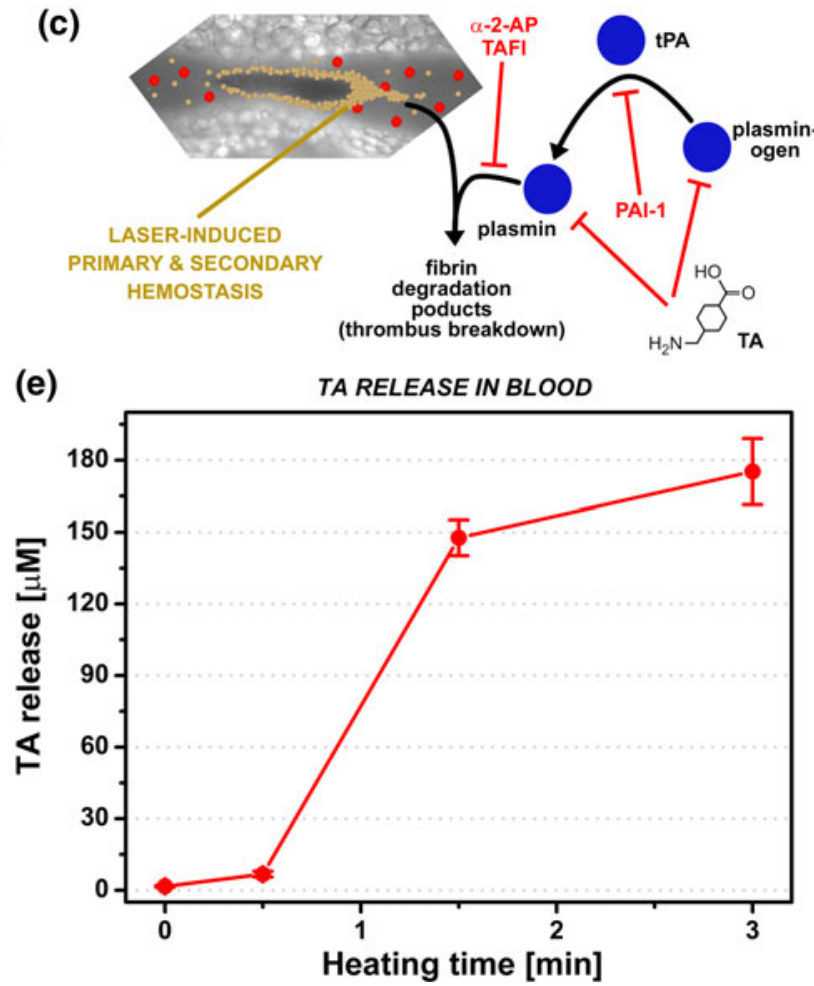

FIGURE 13. (a) Model liposomal DDS for antifibrinolytic site-specific pharmaco-laser therapy, consisting of 1,2-dipalmitoyl-snglycero-3-phosphocholine (DPPC, $96 \mathrm{~mol} \%$ ) and a molar fraction of polyethylene glycol-conjugated 1,2-distearoyl-sn-glycero-3phosphoethanolamine (DSPE-PEG, $4 \mathrm{~mol} \%$ ). The phase transition temperature $\left(T_{\mathrm{m}}\right)$ of this formulation is $42.3^{\circ} \mathrm{C}$, i.e., $\sim 5^{\circ} \mathrm{C}$ above body temperature. The PEG can be used to conjugate antibodies for immunotargeting to the laser-induced thrombus via e.g., Pselectin or fibrin. The liposomes encapsulate tranexamic acid (TA, green circles) in the aqueous compartment. The chemical structure of TA is provided in (b). (c) Mechanism of fibrinolysis and inhibitory pathways in the context of laser-induced thrombosis. Plasminogen is converted to plasmin by tissue-type plasminogen activator (tPA), which cleaves cross-polymerized fibrin strands in the thrombus, resulting in the generation of fibrin degradation products and embolization. Plasminogen activator inhibitor-1 (PAI-1) inhibits the conversion of plasminogen to plasmin, alpha-2 antiplasmin ( $\alpha-2-\mathrm{AP})$ and thrombin-activatable fibrinolysis inhibitor (TAFI) antagonize the enzymatic activity of plasmin, and TA inhibits plasmin(ogen) at the lysine binding sites. The liposomes described in (a) were prepared with $316 \mathrm{mM}$ TA and assayed for heat-induced drug release. (d) Heat-induced release kinetics of TA from thermosensitive liposomes measured near $T_{\mathrm{m}}$ and $4{ }^{\circ} \mathrm{C}$ below $T_{\mathrm{m}}$ (control) in buffer $(10 \mathrm{mM} \mathrm{HEPES}, 0.88 \% \mathrm{NaCl}$, $\mathrm{pH}=7.4,0.292$ osmol $/ \mathrm{kg}$ ). Near-complete TA release was achieved within $2.5 \mathrm{~min}$ of heating at $43.3^{\circ} \mathrm{C}$, whereas heating at $39.3^{\circ} \mathrm{C}$ resulted in $\sim 13 \%$ TA release after 5 min (unpublished results). In (e), heat-induced TA release from thermosensitive liposomes was measured in whole blood, ${ }^{75}$ exhibiting comparable TA release kinetics as in buffer. 
generation photosensitizer, in the lipid bilayer (Figs. 12a and 12b). Photosensitizers are compounds that, upon excitation to triplet state by resonant light, undergo electron transfer (type I) or energy transfer (type II) reactions with molecular oxygen to form ROS (Fig. 12c). The generation of these reactive transients leads to irreversible modification of biomolecules and, when generated in the circulation, to platelet ${ }^{45,46}$ and endothelial cell damage/activation ${ }^{63,101,157}$ and subsequent thrombosis. ${ }^{91,123}$ Consequently, incorporation and photo-activation of $\mathrm{ZnPC}$-liposomes in laser-induced thrombi is expected to result in exacerbation of the hemodynamic response and ultimately complete occlusion of otherwise incompletely photocoagulated vascular lumens. In vitro experiments have revealed that liposomes composed of DPPC:cholesterol:DSPEPEG (66:30:4 mol ratio) encapsulating $\mathrm{ZnPC}$ at a 0.004 photosensitizer:lipid ratio are capable of generating ROS (Fig. 12d), induce perturbations in lipid bilayers (Fig. 12e), and oxidize amino acid residues in proteins (Fig. 12f). These data, albeit preliminary, have established proof-of-concept and support the postulation that hypertrombosis can be achieved with this liposomal DDS by photochemical means.

\section{Liposomes for Antifibrinolytic Site-Specific Pharmaco-Laser Therapy}

Given the thermal nature of endovascular laser-tissue interactions, the triggering mechanism of the rudimentary antifibrinolytic DDS was designed around thermosensitivity. The DDS for antifibrinolytic SSPLT therefore comprises thermosensitive liposomes (Figs. 11b-11 $\mathrm{d}^{102,103}$ ) that encapsulate tranexamic acid (TA) in the aqueous compartment (Fig. 13a). TA is an antifibrinolytic agent (Fig. 13b) that is widely used in the clinical setting for a variety of bleeding-deterrent procedures. ${ }^{78,89,96,128,161}$ TA completely antagonizes the biological activity of plasmin(ogen) by occupying its lysine binding sites $^{135}$ (Fig. 13c). Plasmin, which is gradually formed with the onset of coagulation, is responsible for cleaving cross-polymerized fibrin strands that make up the reticular network of the thrombus. ${ }^{108}$ The absence of fibrinolysis by the inhibitory effect of TA will therefore preserve thrombus integrity and promote thrombus stability during and after laser-induced thrombus formation, delaying gradual thrombus dissolution as a result of fibrinolysis and shear stress.

TA-encapsulating liposomes composed of DPPC:DSPE-PEG in a 96:4 mol ratio have been prepared and characterized. ${ }^{66,67}$ These liposomes exhibit a phase transition maximum at $42.3{ }^{\circ} \mathrm{C}$, which is approximately $5{ }^{\circ} \mathrm{C}$ above body temperature and therefore presumed safe for use in patients with PWSs but without any coagulopathies. Preliminary in vitro experiments have demonstrated that almost complete release of TA from thermosensitive liposomes in buffered solution can be achieved within $2.5 \mathrm{~min}$ of heating near the phase transition temperature $\left(43.3{ }^{\circ} \mathrm{C}\right)$, whereas minimal TA release occurs at temperatures slightly above body temperature $\left(39.3{ }^{\circ} \mathrm{C}\right)$ (Fig. $\left.13 \mathrm{~d}^{66,67}\right)$. Similarly, TA-liposomes suspended in whole blood exhibit similar release kinetics as the buffer-suspended liposomes when heated at $43.3{ }^{\circ} \mathrm{C}$ (Fig. 13e), ${ }^{75}$ indicating that plasma components do not detrimentally impact heat-induced TA release in physiological fluids. ${ }^{37,61,125}$ The current lack of in vivo proof-of-concept notwithstanding, the data provide compelling evidence for the potential efficacy of antifibrinolytic intervention in laser-treated, partially occluded PWS vasculature.

\section{ACKNOWLEDGMENTS}

Bernard Choi and Kristen M. Kelly acknowledge PHS-NIH for financial support through the Child Health \& Human Development Award (R01HD065536, Novel Optical Treatment Approach for Vascular Birthmarks). Some of the data presented were obtained from research projects sponsored by the National Anticancer Foundation (Stichting Nationaal Fonds Tegen Kanker) in Amsterdam (MB, MH).

\section{OPEN ACCESS}

This article is distributed under the terms of the Creative Commons Attribution Noncommercial License which permits any noncommercial use, distribution, and reproduction in any medium, provided the original author(s) and source are credited.

\section{REFERENCES}

\footnotetext{
${ }^{1}$ Adatto, M. A., J. Luc-Levy, and S. Mordon. Efficacy of a novel intense pulsed light system for the treatment of port wine stains. J. Cosmet. Laser Ther. 12:54-60, 2010.

${ }^{2}$ Aguilar, G., W. Franco, J. Liu, L. O. Svaasand, and J. S. Nelson. Effects of hypobaric pressure on human skin: implications for cryogen spray cooling (part II). Laser Surg. Med. 36:130-135, 2005.

${ }^{3}$ Aguilar, G., L. O. Svaasand, and J. S. Nelson. Effects of hypobaric pressure on human skin: feasibility study for port wine stain laser therapy (part 1). Laser Surg. Med. 36:124-129, 2005.

${ }^{4}$ Allen, T. M., C. Hansen, F. Martin, C. Redemann, and A. Yauyoung. Liposomes containing synthetic lipid derivatives of poly(ethylene glycol) show prolonged circulation half-lives in vivo. Biochim. Biophys. Acta 1066:29-36, 1991.
} 
${ }^{5}$ Alster, T. S., and E. L. Tanzi. Combined 595-nm and 1, 064-nm laser irradiation of recalcitrant and hypertrophic port-wine stains in children and adults. Dermatol. Surg. 35:914-919, 2009

${ }^{6}$ Altshuler, G. B., H. H. Zenzie, A. V. Erofeev, M. Z. Smirnov, R. R. Anderson, and C. Dierickx. Contact cooling of the skin. Phys. Med. Biol. 44:1003-1023, 1999.

${ }^{7}$ Anderson, R. R., and J. A. Parrish. Selective photothermolysis: precise microsurgery by selective absorption of pulsed radiation. Science 220:524-527, 1983.

${ }^{8}$ Anvari, B., T. E. Milner, B. S. Tanenbaum, and J. S. A. Nelson. Comparative study of human skin thermal response to sapphire contact and cryogen spray cooling. IEEE Trans. Biomed. Eng. 45:934-941, 1998.

${ }^{9}$ Anvari, B., B. S. Tanenbaum, T. E. Milner, S. Kimel, L. O. Svaasand, and J. S. A. Nelson. Theoretical study of the thermal response of skin to cryogen spray cooling and pulsed laser irradiation: implications for treatment of port wine stain birthmarks. Phys. Med. Biol. 40:1451-1465, 1995.

${ }^{10}$ Anyarambhatla, G. R., and D. Needham. Enhancement of the phase transition permeability of DPPC liposomes by incorporation of MPPC: a new temperature-sensitive liposome for use with mild hyperthermia. J. Liposome Res. 9:491-506, 1999.

${ }^{11}$ Asahina, A., T. Watanabe, A. Kishi, N. Hattori, A. Shirai, S. Kagami, R. Watanabe, A. Le Pavoux, T. Maekawa, K. Tamaka, and K. Ohara. Evaluation of the treatment of port-wine stains with the 595-nm long pulsed dye laser: a large prospective study in adult Japanese patients. $J$. Am. Acad. Dermatol. 54:487-493, 2006.

${ }^{12}$ Ashinoff, R., and R. G. Geronemus. Flashlamp-pumped pulsed dye-laser for port-wine stains in infancy - earlier versus later treatment. J. Am. Acad. Dermatol. 24:467-472, 1991.

${ }^{13}$ Awasthi, V. D., D. Garcia, B. A. Goins, and W. T. Phillips. Circulation and biodistribution profiles of longcirculating PEG-liposomes of various sizes in rabbits. Int J. Pharm. 253:121-132, 2003.

${ }^{14}$ Barsky, S. H., S. Rosen, D. E. Geer, and J. M. Noe. Nature and evolution of port wine stains - computer-assisted study. J. Investig. Dermatol. 74:154-157, 1980.

${ }^{15}$ Barton, J. K., A. Rollins, S. Yazdanfar, T. J. Pfefer, V. Westphal, and J. A. Izatt. Photothermal coagulation of blood vessels: a comparison of high-speed optical coherence tomography and numerical modelling. Phys. Med. Biol. 46:1665-1678, 2001.

${ }^{16}$ Basinger, B., G. Aguilar, and J. S. Nelson. Effect of skin indentation on heat transfer during cryogen spray cooling. Laser Surg. Med. 34:155-163, 2004.

${ }^{17}$ Bassett, J. B., R. U. Anderson, and J. R. Tacker. Use of temperature-sensitive liposomes in the selective delivery of methotrexate and cisplatinum analogs to murine bladder-tumor. J. Urol. 135:612-615, 1986.

${ }^{18}$ Baumler, W., E. Vural, M. Landthaler, F. Muzzi, and G. Shafirstein. The effects of intense pulsed light (IPL) on blood vessels investigated by mathematical modeling. Lasers Surg. Med. 39:132-139, 2007.

${ }^{19}$ Bezemer, R., M. Heger, J. P. H. van den Wijngaard, S. R. Mordon, M. J. C. van Gemert, and J. F. Beek. Laser-induced (endo) vascular photothermal effects studied by combined brightfield and fluorescence microscopy in hamster dorsal skin fold venules. Opt. Express 15:8493$8506,2007$.

${ }^{20}$ Bjerring, P., K. Christiansen, and A. Troilius. Intense pulsed light source for the treatment of dye laser resistant port-wine stains. J. Cosmet. Laser Ther. 5:7-13, 2003.
${ }^{21}$ Black, J. F., and J. K. Barton. Chemical and structural changes in blood undergoing laser photocoagulation. Photochem. Photobiol. 80:89-97, 2004.

${ }^{22}$ Black, J. F., N. Wade, and J. K. Barton. Mechanistic comparison of blood undergoing laser photocoagulation at 532 and 1, 064 nm. Laser Surg. Med. 36:155-165, 2005.

${ }^{23}$ Boas, D. A., and A. K. Dunn. Laser speckle contrast imaging in biomedical optics. J Biomed. Opt. 15:011109, 2010.

${ }^{24}$ Braverman, I. M. The cutaneous microcirculation. J. Investig. Dermatol. Symp. Proc. 5:3-9, 2000.

${ }^{25}$ Bui, A. K., K. M. Teves, E. Indrawan, W. Jia, and B. Choi. Longitudinal, multimodal functional imaging of microvascular response to photothermal therapy. Opt. Lett. 35:3216-3218, 2010.

${ }^{26}$ Campbell, P. I. Toxicity of some charged lipids used in liposome preparations. Cytobios 37:21-26, 1983.

${ }^{27}$ Chan, H. H., E. Chan, T. Kono, S. Y. Ying, and H. WaiSun. The use of variable pulse width frequency doubled $\mathrm{Nd}$ :YAG $532 \mathrm{~nm}$ laser in the treatment of port-wine stain in Chinese patients. Dermatol. Surg. 26:657-661, 2000.

${ }^{28}$ Cheng, H. Y., Y. M. Yan, and T. Q. Duong. Temporal statistical analysis of laser speckle images and its application to retinal blood-flow imaging. Opt. Express 16:10214-10219, 2008.

${ }^{29}$ Childers, M. A., W. Franco, J. S. Nelson, and G. Aguilar. Laser surgery of port wine stains using local vacuum pressure: changes in skin morphology and optical properties (part I). Lasers Surg. Med. 39:108-117, 2007.

${ }^{30}$ Choi, B., W. Jia, J. Channual, K. M. Kelly, and J. Lotfi. The importance of long-term monitoring to evaluate the microvascular response to light-based therapies. J. Invest. Dermatol. 128:485-488, 2008.

${ }^{31}$ Choi, B., B. Majaron, and J. S. Nelson. Computational model to evaluate port wine stain depth profiling using pulsed photothermal radiometry. J. Biomed. Opt. 9:299307, 2004.

${ }^{32}$ Chowdhury, M. M. U., S. Harris, and S. W. Lanigan. Potassium titanyl phosphate laser treatment of resistant port-wine stains. Br. J. Dermatol. 144:814-817, 2001.

${ }^{33}$ Chung, H. H., D. Sun, A. Huang, A. Koller, and G. Kaley. Nitric oxide (NO) release as a function of wall shear stress in isolated rat mesenteric arteries. FASEB J. 12:A79, 1998

${ }^{34}$ Civas, E., E. Koc, B. Aksoy, and H. M. Aksoy. Clinical experience in the treatment of different vascular lesions using a neodymium-doped yttrium aluminum garnet laser. Dermatol. Surg. 35:1933-1941, 2009.

${ }^{35}$ Costanzo, L. S. Physiology. Philadelphia: Saunders/Elsevier, 2003.

${ }^{36}$ Dai, T. H., B. M. Pikkula, J. W. Tunnell, D. W. Chang, and B. Anvari. Thermal response of human skin epidermis to 595-nm laser irradiation at high incident dosages and long pulse durations in conjunction with cryogen spray cooling: an ex vivo study. Laser Surg. Med. 33:16-24, 2003.

${ }^{37}$ Damen, J., J. Regts, and G. Scherphof. Transfer and exchange of phospholipid between small unilamellar liposomes and rat plasma high density lipoproteins. Dependence on cholesterol content and phospholipid composition. Biochim. Biophys. Acta 665:538-545, 1981.

${ }^{38}$ Datrice, N., J. C. Ramirez-San-Juan, R. Zhang, A. Meshkinpour, G. Aguilar, J. S. Nelson, and K. M. Kelly. Cutaneous effects of cryogen spray cooling on in vivo human skin. Dermatol. Surg. 32:1007-1012, 2006. 
${ }^{39}$ Dierickx, C. C., J. M. Casparian, V. Venugopalan, W. A. Farinelli, and R. R. Anderson. Thermal relaxation of port-wine stain vessels probed in vivo - the need for 1-10 millisecond laser-pulse treatment. J. Investig. Dermatol. 105:709-714, 1995.

${ }^{40}$ Duerrschmidt, N., C. Stielow, G. Muller, P. J. Pagano, and $\mathrm{H}$. Morawietz. NO-mediated regulation of $\mathrm{NAD}(\mathrm{P}) \mathrm{H}$ oxidase by laminar shear stress in human endothelial cells. J. Physiol. (Lond) 576:557-567, 2006.

${ }^{41}$ Dummer, R., P. Graf, C. Greif, and G. Burg. Treatment of vascular lesions using the VersaPulse $(R)$ variable pulse width frequency doubled neodymium: YAG laser. Dermatology 197:158-161, 1998.

${ }^{42}$ Duncan, D. D., and S. J. Kirkpatrick. Can laser speckle flowmetry be made a quantitative tool? J Opt. Soc. Am. A Opt. Image Sci Vis. 25:2088-2094, 2008.

${ }^{43}$ Edris, A., B. Choi, G. Aguilar, and J. S. Nelson. Measurements of laser light attenuation following cryogen spray cooling spurt termination. Laser Surg. Med. 32:143147, 2003.

${ }^{44}$ Enjolras, O., and J. B. Mulliken. The current management of vascular birthmarks. Pediatr. Dermatol. 10:311-333, 1993.

${ }^{45}$ Fingar, V. H. Vascular effects of photodynamic therapy. $J$ Clin. Laser Med. Surg. 14:323-328, 1996.

${ }^{46}$ Fingar, V. H., T. J. Wieman, and P. S. Haydon. The effects of thrombocytopenia on vessel stasis and macromolecular leakage after photodynamic therapy using photofrin. Photochem. Photobiol. 66:513-517, 1997.

${ }^{47}$ Fiskerstrand, E. J., L. O. Svaasand, G. Kopstad, M. Dalaker, L. T. Norvang, and G. Volden. Laser treatment of port wine stains: therapeutic outcome in relation to morphological parameters. Br. J. Dermatol. 134:10391043, 1996.

${ }^{48}$ Fiskerstrand, E. J., L. O. Svaasand, G. Kopstad, K. Ryggen, and S. Aase. Photothermally induced vesselwall necrosis after pulsed dye laser treatment: lack of response in port-wine stains with small sized or deeply located vessels. J. Investig. Dermatol. 107:671-675, 1996.

${ }^{49}$ Franco, W., M. Childers, J. Stuart Nelson, and G. Aguilar. Laser surgery of port wine stains using local vaccum pressure: changes in calculated energy deposition (part ii). Lasers Surg. Med. 39:118-127, 2007.

${ }^{50}$ Gaber, M. H., K. L. Hong, S. K. Huang, and D. Papahadjopoulos. Thermosensitive sterically stabilized liposomes - formulation and in vitro studies on mechanism of doxorubicin release by bovine serum and human plasma. Pharm. Res. 12:1407-1416, 1995.

${ }^{51}$ Gaber, M. H., N. Z. Wu, K. L. Hong, S. K. Huang, M. W. Dewhirst, and D. Papahadjopoulos. Thermosensitive liposomes: extravasation and release of contents in tumor microvascular networks. Int. J. Radiat. Oncol. Biol. Phys. 36:1177-1187, 1996.

${ }^{52}$ Ganong, W. F. Review of Medical Physiology. Stamford, CT: Appleton \& Lange, 1997.

${ }^{53}$ Garden, J. M., L. L. Polla, and O. T. Tan. The treatment of port-wine stains by the pulsed dye-laser-analysis of pulse duration and long-term therapy. Arch. Dermatol. 124:889-896, 1988.

${ }^{54}$ Gerasimov, O. V., J. A. Boomer, M. M. Qualls, and D. H. Thompson. Cytosolic drug delivery using ph- and lightsensitive liposomes. Adv. Drug Deliv. Rev. 38:317-338, 1999.

${ }^{55}$ Geronemus, R. G., and R. Ashinoff. The medical necessity of evaluation and treatment of port-wine stains. $J$. Dermatol. Surg. Oncol. 17:76-79, 1991.
${ }^{56}$ Geronemus, R. G., A. T. Quintana, W. W. Lou, and A. N. Kauvar. High-fluence modified pulsed dye laser photocoagulation with dynamic cooling of port-wine stains in infancy. Arch. Dermatol. 136:942-943, 2000.

${ }^{57}$ Goh, C. L. Treatment response of port-wine stains with the flashlamp-pulsed dye laser in the national skin centre: a report of 36 patients. Ann. Acad. Med. Singap. 25:536$540,1996$.

${ }^{58}$ Goh, C. L. Flashlamp-pumped pulsed dye laser (585 nm) for the treatment of portwine stains - a study of treatment outcome in 94 Asian patients in Singapore. Singap. Med. J. 41:24-28, 2000.

${ }^{59}$ Goldman, M. P., R. E. Fitzpatrick, and J. Ruizesparza. Treatment of port-wine stains (capillary malformation) with the flashlamp-pumped pulsed dye-laser. J. Pediatr. 122:71-77, 1993.

${ }^{60}$ Greve, B., and C. Raulin. Prospective study of port wine stain treatment with dye laser: comparison of two wavelengths (585 nm vs. $595 \mathrm{~nm}$ ) and two pulse durations (0.5 milliseconds vs. 20 milliseconds). Laser Surg. Med. 34:168-173, 2004.

${ }^{61}$ Guo, L. S., R. L. Hamilton, J. Goerke, J. N. Weinstein, and R. J. Havel. Interaction of unilamellar liposomes with serum lipoproteins and apolipoproteins. J. Lipid Res. 21:993-1003, 1980.

${ }^{62}$ Hansen, C. B., G. Y. Kao, E. H. Moase, S. Zalipsky, and T. M. Allen. Attachment of antibodies to sterically stabilized liposomes - evaluation, comparison and optimization of coupling procedures. Biochim. Biophys. Acta Biomembr. 1239:133-144, 1995.

${ }^{63}$ He, D. P., J. A. Hampton, R. Keck, and S. H. Selman. Photodynamic therapy: effect on the endothelial cell of the rat aorta. Photochem. Photobiol. 54:801-804, 1991.

${ }^{64}$ Heger, M., J. F. Beek, N. I. Moldovan, C. M. A. M. van der Horst, and M. J. C. van Gemert. Towards optimization of selective photothermolysis: prothrombotic pharmaceutical agents as potential adjuvants in laser treatment of port wine stains - a theoretical study. Thromb. Haemost. 93:242-256, 2005.

${ }^{65}$ Heger, M., R. Bezemer, J. F. Huertas-Perez, H. Dekker, and J. F. Beek. Endovascular laser-tissue interactions redefined: shining light on novel windows of therapeutic opportunity beyond selective photothermolysis. Photomed. Laser Surg. 28:569-572, 2010.

${ }^{66}$ Heger, M., and A. I. P. M. de Kroon. Tranexamic acidcontaining liposomes for antifibrinolytic site-specific pharmaco-laser therapy of port wine stains. Laser Surg. Med. 43:919, 2011.

${ }^{67}$ Heger, M., I. I. Salles, R. Bezemer, S. Mordon, S. Begu, N. Jouy, H. Deckmyn, M. J. C. van Gemert, and J. F. Beek. Thrombosis as an integral part of endovascular laser-tissue interactions. Laser Surg. Med. Suppl 18:5, 2006.

${ }^{68}$ Heger, M., I. Salles, R. Bezemer, et al. Laser-induced primary and secondary hemostasis dynamics and mechanisms in relation to selective photothermolysis of port wine stains. J. Dermatol. Sci. 63:139-147, 2011.

${ }^{69}$ Heger, M., I. I. Salles, W. van Vuure, H. Deckmyn, and J. F. Beek. Fluorescent labeling of platelets with polyanionic fluorescein derivatives. Anal. Quant. Cytol. Histol. 31:227-232, 2009.

${ }^{70}$ Ho, W. S., H. H. Chan, S. Y. Ying, and P. C. Chan. Laser treatment of congenital facial port-wine stains: long-term efficacy and complication in Chinese patients. Laser Surg. Med. 30:44-47, 2002. 
${ }^{71}$ Ho, W. S., S. Y. Ying, P. C. Chan, and H. H. Chan. Treatment of port wine stains with intense pulsed light: a prospective study. Dermatol. Surg. 30:887-891, 2004.

${ }^{72}$ Hohenleutner, U., M. Hilbert, U. Wlotzke, and M. Landthaler. Epidermal damage and limited coagulation depth with the flashlamp-pumped pulsed dye-laser-a histochemical-study. J. Investig. Dermatol. 104:798-802, 1995.

${ }^{73}$ Huang, Y. C., T. L. Ringold, J. S. Nelson, and B. Choi. Noninvasive blood flow imaging for real-time feedback during laser therapy of port wine stain birthmarks. Lasers Surg. Med. 40:167-173, 2008.

${ }^{74}$ Huang, Y. C., N. Tran, P. R. Shumaker, K. Kelly, E. V. Ross, J. S. Nelson, and B. Choi. Blood flow dynamics after laser therapy of port wine stain birthmarks. Lasers Surg. Med. 41:563-571, 2009.

${ }^{75}$ Huertas-Perez, J. F., M. Heger, H. Dekker, H. Krabbe, J. Lankelma, and F. Ariese. Simple, rapid, and sensitive liquid chromatography-fluorescence method for the quantification of tranexamic acid in blood. J. Chromatogr. A 1157:142-150, 2007.

${ }^{76}$ Jia, W., V. Sun, N. Tran, B. Choi, S. W. Liu, M. C. Mihm, Jr., T. L. Phung, and J. S. Nelson. Long-term blood vessel removal with combined laser and topical rapamycin antiangiogenic therapy: implications for effective port wine stain treatment. Lasers Surg. Med. 42:105-112, 2010.

${ }^{77}$ Kao, B., K. M. Kelly, G. Aguilar, Y. Hosaka, R. J. Barr, and J. S. Nelson. Evaluation of cryogen spray cooling exposure on in vitro model human skin. Laser Surg. Med. 34:146-154, 2004.

${ }^{78}$ Karski, J., G. Diaiani, J. Carroll, M. Lwanochko, P. Seneviratne, P. Liu, W. Kucharczyk, L. Fedorko, T. David, and D. Cheng. Tranexamic acid and early saphenous vein graft patency in conventional coronary artery bypass graft surgery: a prospective randomized controlled clinical trial. J. Thorac. Cardiovasc. Surg. 130:309-314, 2005.

${ }^{79}$ Kelly, K. M., B. Choi, S. McFarlane, A. Motosue, B. Jung, M. H. Khan, J. C. Ramirez-San-Juan, and J. S. Nelson. Description and analysis of treatments for portwine stain birthmarks. Arch. Facial Plast. Surg. 7:287294, 2005.

${ }^{80}$ Kelly, K. M., V. S. Nanda, and J. S. Nelson. Treatment of port-wine stain birthmarks using the 1.5 -msec pulsed dye laser at high fluences in conjunction with cryogen spray cooling. Dermatol. Surg. 28:309-313, 2002.

${ }^{81}$ Klibanov, A. L., K. Maruyama, V. P. Torchilin, and L. Huang. Amphipathic polyethyleneglycols effectively prolong the circulation time of liposomes. FEBS Lett. 268:235-237, 1990.

${ }^{82}$ Kong, G., and M. W. Dewhirst. Hyperthermia and liposomes. Int. J. Hyperth. 15:345-370, 1999.

${ }^{83}$ Kono, T., W. F. Groff, H. H. Chan, H. Sakurai, and T. Yamaki. Long-pulsed neodymium:yttrium-aluminumgarnet laser treatment for hypertrophic port-wine stains on the lips. J. Cosmet. Laser Ther. 11:11-13, 2009.

${ }^{84}$ Kono, T., H. Sakurai, M. Takeuchi, T. Yamaki, K. Soejima, W. F. Groff, and M. Nozaki. Treatment of resistant port-wine stains with a variable-pulse pulsed dye laser. Dermatol. Surg. 33:951-956, 2007.

${ }^{85}$ Lakmaker, O., J. W. Pickering, and M. J. C. van Gemert. Modeling the color-perception of port wine stains and its relation to the depth of laser coagulated blood-vessels. Laser Surg. Med. 13:219-226, 1993.

${ }^{86}$ Lanigan, S. W., and J. A. Cotterill. Psychological disabilities amongst patients with port wine stains. Br. J. Dermatol. 121:209-215, 1989.
${ }^{87}$ Lasic, D. D. Liposomes. Sci. Med. 3:34-43, 1996.

${ }^{88}$ Laube, S., S. Taibjee, and S. W. Lanigan. Treatment of resistant port wine stains with the v beam (r) pulsed dye laser. Laser Surg. Med. 33:282-287, 2003.

${ }^{89}$ Levy, J. H. Pharmacologic preservation of the hemostatic system during cardiac surgery. Ann. Thorac. Surg. 72:S1814-S1820, 2001.

${ }^{90} \mathrm{Li}$, G., T. Lin, Q. J. Wu, Z. C. Zhou, and M. H. Gold. Clinical analysis of port wine stains treated by intense pulsed light. J. Cosmet. Laser Ther. 12:2-6, 2010.

${ }^{91}$ Lindberg, R. A., D. W. Slaaf, A. B. Lentsch, and F. N. Miller. Involvement of nitric oxide and cyclooxygenase products in photoactivation-induced microvascular occlusion. Microvasc. Res. 47:203-221, 1994.

${ }^{92}$ Lucassen, G. W., W. Verkruysse, M. Keijzer, and M. J. C. van Gemert. Light distributions in a port wine stain model containing multiple cylindrical and curved blood vessels. Laser Surg. Med. 18:345-357, 1996.

${ }^{93}$ Majaron, B., S. Kimel, W. Verkruysse, G. Aguilar, R. Pope, L. O. Svaasand, E. J. Lavernia, and J. S. Nelson. Cryogen spray cooling in laser dermatology: effects of ambient humidity and frost formation. Laser Surg. Med. 28:469-476, 2001.

${ }^{94}$ Majaron, B., W. Verkruysse, B. S. Tanenbaum, T. E. Milner, and J. S. Nelson. Spectral variation of the infrared absorption coefficient in pulsed photothermal profiling of biological samples. Phys. Med. Biol. 47:1929-1946, 2002.

${ }^{95}$ Majaron, B., W. Verkruysse, B. S. Tanenbaum, T. E. Milner, S. A. Telenkov, D. M. Goodman, and J. S. Nelson. Combining two excitation wavelengths for pulsed photothermal profiling of hypervascular lesions in human skin. Phys. Med. Biol. 45:1913-1922, 2000.

${ }^{96}$ Mannucci, P. M. Drug therapy-treatment of Von Willebrand's disease. N. Engl. J. Med. 351:683-694, 2004.

${ }^{97}$ Maruyama, K., S. Unezaki, N. Takahashi, and M. Iwatsuru. Enhanced delivery of doxorubicin to tumor by long-circulating thermosensitive liposomes and local hyperthermia. Biochim. Biophys. Acta 1149:209-216, 1993.

${ }^{98}$ Mayhew, E., M. Ito, and R. Lazo. Toxicity of non-drugcontaining liposomes for cultured human-cells. Exp. Cell Res. 171:195-202, 1987.

${ }^{99}$ Merlin, J. L. In vitro evaluation of the association of thermosensitive liposome-encapsulated doxorubicin with hyperthermia. Eur. J. Cancer 27:1031-1034, 1991.

${ }^{100}$ Milner, T. E., D. M. Goodman, B. S. Tanenbaum, and J. S. Nelson. Depth profiling of laser-heated chromophores in biological tissues by pulsed photothermal radiometry. J. Opt. Soc. Am. A Opt. Image Sci. Vis. 12:14791488, 1995.

${ }^{101}$ Morcos, N. C., F. Zaldivar, H. M. Lo, and W. L. Henry. Bovine coronary artery endothelium: culture, characterization, angiogenesis and sensitivity to laser photodynamic treatment modalities. J Clin. Lab. Immunol. 34:99-106, 1991.

${ }^{102}$ Nagle, J. F. Theory of the main lipid bilayer phase-transition. Annu. Rev. Phys. Chem. 31:157-195, 1980.

${ }^{103}$ Needham, D., and M. W. Dewhirst. The development and testing of a new temperature-sensitive drug delivery system for the treatment of solid tumors. Adv. Drug Deliv. Rev. 53:285-305, 2001.

${ }^{104}$ Nelson, J. S., K. M. Kelly, Y. Zhao, and Z. Chen. Imaging blood flow in human port-wine stain in situ and in real time using optical Doppler tomography. Arch. Dermatol. 137:741-744, 2001.

${ }^{105}$ Nelson, J. S., T. E. Milner, B. Anvari, B. S. Tanenbaum, S. Kimel, L. O. Svaasand, and S. L. Jacques. Dynamic 
epidermal cooling during pulsed laser treatment of portwine stain. A new methodology with preliminary clinical evaluation. Arch. Dermatol. 131:695-700, 1995.

${ }^{106}$ Nguyen, C. M., J. J. Yohn, C. Huff, W. L. Weston, and J. G. Morelli. Facial port wine stains in childhood: prediction of the rate of improvement as a function of the age of the patient, size and location of the port wine stain and the number of treatments with the pulsed dye $(585 \mathrm{~nm})$ laser. Br. J. Dermatol. 138:821-825, 1998.

${ }^{107}$ Nilsson, A. M. K., G. W. Lucassen, W. Verkruysse, S. AnderssonEngels, and M. J. C. van Gemert. Changes in optical properties of human whole blood in vitro due to slow heating. Photochem. Photobiol. 65:366-373, 1997.

${ }^{108}$ Oliver, J. J., D. J. Webb, and D. E. Newby. Stimulated tissue plasminogen activator release as a marker of endothelial function in humans. Arterioscler. Thromb. Vasc. Biol. 25:2470-2479, 2005.

${ }^{109}$ Orten, S. S., M. Waner, S. Flock, P. K. Roberson, and J. Kincannon. Port-wine stains - an assessment of 5 years of treatment. Arch. Otolaryngol. Head Neck Surg. 122:11741179, 1996.

${ }^{110}$ Ozdemir, M., B. Engin, and I. Mevlitoglu. Treatment of facial port-wine stains with intense pulsed light: a prospective study. J. Cosmet. Dermatol. 7:127-131, 2008.

${ }^{111}$ Ozkucur, N., E. Richter, C. Wetzel, F. Hollstein, R. H. W. Funk, and T. K. Monsees. Effect of shear stress on nitric oxide (NO) production in human primary endothelial cells grown on modified polyurethane (PUR) surfaces. Tissue Eng. 13:919, 2007

${ }^{112}$ Papahadjopoulos, D., T. M. Allen, A. Gabizon, E. Mayhew, K. Matthay, S. K. Huang, K. D. Lee, M. C. Woodle, D. D. Lasic, C. Redemann, and F. J. Martin. Sterically stabilized liposomes - improvements in pharmacokinetics and antitumor therapeutic efficacy. Proc. Natl. Acad. Sci. U.S.A. 88:11460-11464, 1991.

${ }^{113}$ Pence, B., B. Aybey, and G. Ergenekon. Outcomes of 532 $\mathrm{nm}$ frequency-doubled Nd: YAG laser use in the treatment of port-wine stains. Dermatol. Surg. 31:509-517, 2005.

${ }^{114}$ Pfefer, T. J., J. K. Barton, D. J. Smithies, T. E. Milner, J. S. Nelson, M. J. C. van Gemert, and A. J. Welch. Modeling laser treatment of port wine stains with a computer-reconstructed biopsy. Laser Surg. Med. 24:151$166,1999$.

${ }^{115}$ Pfefer, T. J., J. K. Barton, D. J. Smithies, T. E. Milner, J. S. Nelson, M. J. van Gemert, and A. J. Welch. Modeling laser treatment of port wine stains with a computerreconstructed biopsy. Lasers Surg. Med. 24:151-166, 1999.

${ }^{116}$ Phung, T. L., D. A. Oble, W. Jia, L. E. Benjamin, M. C. Mihm, Jr., and J. S. Nelson. Can the wound healing response of human skin be modulated after laser treatment and the effects of exposure extended? Implications on the combined use of the pulsed dye laser and a topical angiogenesis inhibitor for treatment of port wine stain birthmarks. Lasers Surg. Med. 40:1-5, 2008.

${ }^{117}$ Pickering, J. W., and M. J. C. van Gemert. $585 \mathrm{~nm}$ for the laser treatment of port wine stains - a possible mechanism. Laser Surg. Med. 11:616-618, 1991.

${ }^{118}$ Qiu, J., P. Li, W. Luo, J. Wang, H. Zhang, and Q. Luo. Spatiotemporal laser speckle contrast analysis for blood flow imaging with maximized speckle contrast. $J$ Biomed. Opt. 15:016003, 2010.

${ }^{119}$ Ramirez-San-Juan, J. C., G. Aguilar, A. Tuqan, K. M. Kelly, and J. S. Nelson. Sub-zero and residence times of multiple cryogen spurts. Laser Surg. Med. 36:141-146, 2004.
${ }^{120}$ Ramirez-San-Juan, J. C., B. Choi, W. Franco, J. S. Nelson, and G. Aguilar. Effect of ambient humidity on light transmittance through skin phantoms during cryogen spray cooling. Phys. Med. Biol. 51:113-120, 2006.

${ }^{121}$ Ramirez-San-Juan, J. C., R. Ramos-Garcia, I. Guizar-Iturbide, G. Martinez-Niconoff, and B. Choi. Impact of velocity distribution assumption on simplified laser speckle imaging equation. Opt. Express 16:3197-3203, 2008.

${ }^{122}$ Raulin, C., and H. Grema. Single-pass carbon dioxide laser skin resurfacing combined with cold-air cooling: efficacy and patient satisfaction of a prospective side-byside study. Arch. Dermatol. 140:1333-1336, 2004.

${ }^{123}$ Reed, M. W., T. J. Wieman, D. A. Schuschke, M. T. Tseng, and F. N. A. Miller. Comparison of the effects of photodynamic therapy on normal and tumor blood vessels in the rat microcirculation. Radiat. Res. 119:542-552, 1989.

${ }^{124}$ Reyes, B. A., and R. Geronemus. Treatment of port-wine stains during childhood with the flashlamp-pumped pulsed dye-laser. J. Am. Acad. Dermatol. 23:1142-1148, 1990.

${ }^{125}$ Scherphof, G., H. Morselt, J. Regts, and J. C. Wilschut. The involvement of the lipid phase transition in the plasma-induced dissolution of multilamellar phosphatidylcholine vesicles. Biochim. Biophys. Acta 556:196-207, 1979.

${ }^{126}$ Selim, M. M., K. M. Kelly, J. S. Nelson, G. Wendelschafer-Crabb, W. R. Kennedy, and B. D. Zelickson. Confocal microscopy study of nerves and blood vessels in untreated and treated port wine stains: preliminary observations. Dermatol. Surg. 30:892-897, 2004.

${ }^{127}$ Senior, J., C. Delgado, D. Fisher, C. Tilcock, and G. Gregoriadis. Influence of surface hydrophilicity of liposomes on their interaction with plasma-protein and clearance from the circulation studies with poly(ethylene glycol)-coated vesicles. Biochim. Biophys. Acta 1062:77$82,1991$.

${ }^{128}$ Sethna, N. F., D. Zurakowski, R. M. Brustowicz, J. Bacsik, L. J. Sullivan, and F. Shapiro. Tranexamic acid reduces intraoperative blood loss in pediatric patients undergoing scoliosis surgery. Anesthesiology 102:727-732, 2005.

${ }^{129}$ Shahinian, S., and J. R. Silvius. A novel strategy affords high-yield coupling of antibody fab' fragments to liposomes. Biochim. Biophys. Acta Biomembr. 1239:157-167, 1995.

${ }^{130}$ Sharma, V. K., and S. Khandpur. Efficacy of pulsed dye laser in facial port-wine stains in Indian patients. Dermatol. Surg. 33:560-566, 2007.

${ }^{131}$ Shum, P., J. M. Kim, and D. H. Thompson. Phototriggering of liposomal drug delivery systems. Adv. Drug Deliv. Rev. 53:273-284, 2001.

${ }^{132}$ Smithies, D. J., M. J. van Gemert, M. K. Hansen, T. E. Milner, and J. S. Nelson. Three-dimensional reconstruction of port wine stain vascular anatomy from serial histological sections. Phys. Med. Biol. 42:1843-1847, 1997.

${ }^{133}$ Sommer, S., and R. A. Sheehan-Dare. Pulsed dye laser treatment of port-wine stains in pigmented skin. J. Am. Acad. Dermatol. 42:667-671, 2000.

${ }^{134}$ Svaasand, L. O., L. T. Norvang, E. J. Fiskerstrand, E. K. S. Stopps, M. W. Berns, and J. S. Nelson. Tissue parameters determining the visual appearance of normal skin and port-wine stains. Laser Med. Sci. 10:55-65, 1995.

${ }^{135}$ Takada, A., and Y. Takada. Inhibition by tranexamic acid of the conversion of single-chain tissue plasminogen-activator to its 2 chain form by plasmin - the presence on 
tissue plasminogen-activator of a site to bind with lysine binding-sites of plasmin. Thromb. Res. 55:717-725, 1989.

${ }^{136}$ Tallman, B., O. T. Tan, J. G. Morelli, J. Piepenbrink, T. J. Stafford, S. Trainor, and W. L. Weston. Location of portwine stains and the likelihood of ophthalmic and or centralnervous-system complications. Pediatrics 87:323-327, 1991.

${ }^{137}$ Tan, O. T., J. M. Carney, R. Margolis, Y. Seki, J. Boll, R. R. Anderson, and J. A. Parrish. Histologic responses of port-wine stains treated by argon, carbon-dioxide, and tunable dye-lasers - a preliminary-report. Arch. Dermatol. 122:1016-1022, 1986

${ }^{138}$ Tan, O. T., J. G. Morelli, D. Whitaker, J. Boll, and G. Murphy. Ultrastructural-changes in red blood-cells following pulsed irradiation in vitro. J. Investig. Dermatol. 92:100-104, 1989.

${ }^{139}$ Tan, O. T., P. Morrison, and A. K. Kurban. 585-nm for the treatment of port-wine stains. Plast. Reconstr. Surg. 86:1112-1117, 1990.

${ }^{140}$ Tan, O. T., D. Whitaker, J. M. Garden, and G. Murphy. Pulsed dye laser $(577 \mathrm{~nm})$ treatment of portwine stains: ultrastructural evidence of neovascularization and mast cell degranulation in healed lesions. J. Invest. Dermatol. 90:395-398, 1988

${ }^{141}$ Torchilin, V. P., A. L. Klibanov, L. Huang, S. Odonnell, N. D. Nossiff, and B. A. Khaw. Targeted accumulation of polyethylene glycol-coated immunoliposomes in infarcted rabbit myocardium. FASEB J. 6:2716-2719, 1992.

${ }^{142}$ Torchilin, V. P., J. Narula, E. Halpern, and B. A. Khaw. Poly(ethylene glycol)-coated anti-cardiac myosin immunoliposomes: factors influencing targeted accumulation in the infarcted myocardium. Biochim. Biophys. Acta Biomembr. 1279:75-83, 1996.

${ }^{143}$ Torres, J. H., J. W. Tunnell, B. M. Pikkula, and B. Anvari. An analysis of heat removal during cryogen spray cooling and effects of simultaneous airflow application. Lasers Surg. Med. 28:477-486, 2001.

${ }^{144}$ Troilius, A., and B. Ljunggren. Reflectance spectrophotometry in the objective assessment of dye laser-treated port-wine stains. Br. J. Dermatol. 132:245-250, 1995.

${ }^{145}$ Troilius, A., G. Svendsen, and B. Ljunggren. Ultrasound investigation of port wine stains. Acta Dermato-Venereol. 80:196-199, 2000.

${ }^{146}$ Tunnell, J. W., D. W. Chang, C. Johnston, J. H. Torres, C. W. Patrick, M. J. Miller, S. L. Thomsen, and B. Anvari. Effects of cryogen spray cooling and high radiant exposures on selective vascular injury during laser irradiation of human skin. Arch. Dermatol. 139:743-750, 2003.

${ }^{147}$ Tunnell, J. W., L. V. Wang, and B. Anvari. Optimum pulse duration and radiant exposure for vascular laser therapy of dark port-wine skin: a theoretical study. Appl. Opt. 42:1367-1378, 2003.

${ }^{148}$ van Gemert, M. J. C., J. S. Nelson, T. E. Milner, D. J. Smithies, W. Verkruysse, J. F. de Boer, G. W. Lucassen, D. M. Goodman, B. S. Tanenbaum, L. T. Norvang, and L. O. Svaasand. Non-invasive determination of port wine stain anatomy and physiology for optimal laser treatment strategies. Phys. Med. Biol. 42:937-950, 1997.
${ }^{149}$ van Gemert, M. J. C., D. J. Smithies, W. Verkruysse, T. E. Milner, and J. S. Nelson. Wavelengths for port wine stain laser treatment: influence of vessel radius and skin anatomy. Phys. Med. Biol. 42:41-50, 1997.

${ }^{150}$ Verkruysse, W., J. F. Beek, E. Van Bavel, M. J. C. van Gemert, and J. A. E. Spaan. Laser pulse impact on rat mesenteric blood vessels in relation to laser treatment of port wine stain. Laser Surg. Med. 28:461-468, 2001.

${ }^{151}$ Verkruysse, W., B. Choi, J. R. Zhang, J. Kim, and J. S. Nelson. Thermal depth profiling of vascular lesions: automated regularization of reconstruction algorithms. Phys. Med. Biol. 53:1463-1474, 2008.

${ }^{152}$ Verkruysse, W., G. W. Lucassen, J. F. de Boer, D. J. Smithies, J. S. Nelson, and M. J. C. van Gemert. Modelling light distributions of homogeneous versus discrete absorbers in light irradiated turbid media. Phys. Med. Biol. 42:51-65, 1997.

${ }^{153}$ Verkruysse, W., G. W. Lucassen, and M. J. C. van Gemert. Simulation of color of port wine stain skin and its dependence on skin variables. Laser Surg. Med. 25:131139, 1999.

${ }^{154}$ Verkruysse, W., J. W. Pickering, J. F. Beek, M. Keijzer, and M. J. C. Van Gemert. Modeling the effect of wavelength on the pulsed dye-laser treatment of port wine stains. Appl. Opt. 32:393-398, 1993.

${ }^{155}$ Verkruysse, W., M. J. C. van Gemert, D. J. Smithies, and J. S. Nelson. Modelling multiple laser pulses for port wine stain treatment. Phys. Med. Biol. 45:N197-N203, 2000.

${ }^{156}$ Verkruysse, W., R. Zhang, B. Choi, G. Lucassen, L. O. Svaasand, and J. S. A. Nelson. Library based fitting method for visual reflectance spectroscopy of human skin. Phys. Med. Biol. 50:57-70, 2005.

${ }^{157}$ Volanti, C., J. Y. Matroule, and J. Piette. Involvement of oxidative stress in NF-kappaB activation in endothelial cells treated by photodynamic therapy. Photochem. Photobiol. 75:36-45, 2002.

${ }^{158}$ Weinstein, J. N., R. L. Magin, R. L. Cysyk, and D. S. Zaharko. Treatment of solid L1210 murine tumors with local hyperthermia and temperature-sensitive liposomes containing methotrexate. Cancer Res. 40:1388-1395, 1980.

${ }^{159}$ Whang, K. K., J. Y. Byun, and S. H. A. Kim. Dualwavelength approach with $585-\mathrm{nm}$ pulsed-dye laser and 800-nm diode laser for treatment-resistant port-wine stains. Clin. Exp. Dermatol. 34:e436-e437, 2009.

${ }^{160}$ Woo, S. H., H. H. Ahn, S. N. Kim, and Y. C. Kye. Treatment of vascular skin lesions with the variable pulse $595 \mathrm{~nm}$ pulsed dye laser. Dermatol. Surg. 32:41-48, 2006.

${ }^{161}$ Yamasaki, S., K. Masuhara, and T. Fuji. Tranexamic acid reduces postoperative blood loss in cementless total hip arthroplasty. J. Bone Joint Surg. Am. 87A:766-770, 2005.

${ }^{162}$ Yang, O., D. Cuccia, and B. Choi. Real-time blood flow visualization using the graphics processing unit. J Biomed. Opt. 16:016009, 2011.

${ }^{163}$ Yeo, C., T. Son, J. Park, Y. H. Lee, K. Kwon, J. S. Nelson, and B. Jung. Development of compression-controlled low-level laser probe system: towards clinical application. Lasers Med. Sci. 25:699-704, 2010. 